\title{
Estimation of combining ability and superiority percentage of half diallel crosses between yellow maize inbred lines for growth characters and some diseases resistance
}

\author{
El-Refaey R.A. ${ }^{1}$, A.A. Motawei $^{2}$, A.A. El-Gammal ${ }^{1}$ and M.S. Kotp ${ }^{2}$ \\ Crop Sci. Dep., Faculty of Agric.,Tanta University, Egypt ${ }^{1}$ \\ Maize Research Department, Sakha ARS, FCRI, ARC, Egypt ${ }^{2}$
}

\section{ABSTRACT}

Half diallel cross among ten yellow maize inbred lines was made at Sakha Agriculture Research Station during the growing season 2015. The resultant 45 crosses along with two commercial hybrids as yellow checks (single crosses 162 and 168) were evaluated at two field experiments in two years 2016 and 2017. The results indicated that, years $(\mathrm{Y})$ mean squares had significant effects for all traits, except resistance to downy mildew disease. Genotypes $(\mathrm{G})$ and crosses $(\mathrm{Cr})$ mean squares had high significant effects for all traits in the two years and its combined data. However, G x Y and $\mathrm{Cr} \times \mathrm{Y}$ interaction were significant or highly significant for all traits. Mean squares due to general combining ability (GCA) and specific combining ability (SCA) were highly significant for all traits in the two years and their combined analysis. On the other side, the interactions between GCA and SCA with years were highly significant for all traits. For GCA / SCA and GCA x Y / SCA x Y mean squares ratio, the data pointed out that, this ratio exceeded the unity for all studied traits in two years and their combined. The inbred line $\mathrm{P}_{4}$ had desirable significant of $\left(\mathrm{g}_{\mathrm{i}}\right)$ for earliness, good plant type, resistance to downy mildew and late wilt diseases, also the inbred line $\mathrm{P}_{8}$ had desirable significant of $\left(\mathrm{g}_{\mathrm{i}}^{\wedge}\right)$ for earliness and good plant type. The single crosses; $\mathrm{P}_{1} \times \mathrm{P}_{3}, \mathrm{P}_{6} \times \mathrm{P}_{7}$ and $\mathrm{P}_{4} \times \mathrm{P}_{5}$ had negative and significant values of $\left(\mathrm{s}_{\mathrm{ij}}\right)$ for good plant type and earliness. The single crosses $\mathrm{P}_{1} \times$ $\mathrm{P}_{2}, \mathrm{P}_{1} \times \mathrm{P}_{8}, \mathrm{P}_{2} \times \mathrm{P}_{8}, \mathrm{P}_{4} \times \mathrm{P}_{8}, \mathrm{P}_{5} \times \mathrm{P}_{8}, \mathrm{P}_{6} \times \mathrm{P}_{8}, \mathrm{P}_{7} \times \mathrm{P}_{8}, \mathrm{P}_{8} \times \mathrm{P}_{9}$ and $\mathrm{P}_{8} \times \mathrm{P}_{10}$ had superiority percentage in desired direction over both check cultivars i.e., SC 162 and SC 168 for earliness and good plant type.

Key words: combining ability, diallel analysis, maize, downy mildew and late wilt diseases.

\section{INTRODUCTION}

Breeding for high yielding ability and resistance to main disease, especially late wilt disease caused by Cephalosporium maydis and downy mildew caused by Peronosclerospora sorgi are considered among the main targets of National Maize Breeding Program in Egypt, to reduce feed and food crisis the amount of imported yellow maize grains. The most efficient, effective, environmentally safe and economic means to control late wilt and downy mildew diseases of maize is to use resistant varieties.

Late wilt is one of the major diseases affecting maize in Egypt. Among tests to evaluate hybrids in Egypt is evaluation for yield potentiality and resistance to late wilt under artificial infection. Samra et al. (1962) were the first isolated the pathogen from the roots and stems of wilting maize plants in 1960 and identified the late wilt pathogen as Cephalosporium maydis. Sabet et al. (1970) reported that C. maydis infected young plants easily but when the plants aged, few plants were infected and none infected after approximately 50 days from sowing. The information of the type of gene action that control resistance is very important for breeders in planning the most appropriate and effective breeding method to improve the trait. El-Itriby et al. (1984) and Amer et al. (2002) reported that, dominance and epistasis were the major contributors to inheritance of resistance to late wilt. Galal $\boldsymbol{e t}$ al. (1979), Nawar and Salem (1985) and Mosa and Motawei (2005) indicated that, the additive gene effects played the major role in the expression of the resistance to the late wilt.

Sorghum downy mildew (SDM) is one of the most destructive diseases of maize in Egypt, especially in Delta region. The symptoms of SDM are characterized in that leaves of infected plants are narrower and more erect than those of healthy plants and diseased plants may have phylloddied tassels. In 1928 growing season, SDM disease was first identified on maize sowings adjacent to sorghum fields in Egypt (Melchers, 1931) at Giza Agricultural experimental farm belonging to the Ministry of Agriculture. The SDM disease was not listed as serious disease for either sorghum or maize in Egypt until 1975 (Salama, 1976). Since that time, this disease is of economic importance. In the last few years, SDM disease started to be one of the serious diseases on maize in Egypt, especially in Delta region as a result of wide spread of sorghum sowings in this region, which is considered the main host plant and spreader of the $P$. sorghi. Maize is also considered the second host of the downy mildew pathogen. Therefore, greater efforts are attempted by Egyptian maize breeders to look for 
sources of resistance to this disease and to transfer the resistance to the commercial hybrids and varieties. Knowledge of the type of gene action of maize resistance is necessary in planning the most appropriate and effective breeding program to improve the trait. El-Shenawy (1995), Amer et al. (2002) and Nair et al. (2004) found that, the additive gene effects played the major role in the expression of resistance to the downy mildew. While, Kaneko and Aday (1980) and Mosa et al. (2009) reported that, the non-additive gene action was important in controlling the behavior of resistance to downy mildew disease.

Diallel crosses have been widely used in genetic research to determine general and specific combining abilities (GCA and SCA) and nature of gene action for many traits. Analysis of diallel data is usually conducted according to the methods of Griffing (1956) which partition the total variation of diallel data into GCA of the parents and SCA of the crosses. Combining ability information is necessary for selection of suitable advanced lines for hybridization and identification of promising hybrids.

The main objectives of the present work are to identify the type of gene action that control the inheritance of all studied traits, estimate of general combining ability effects for ten yellow inbred lines and specific combining ability effects for their crosses and estimate the percentage of superiority for diseases resistance and some maize growth characters.

\section{MATERIALS AND METHODS}

This investigation was carried out at Sakha Agriculture Research Stations, Agric. Res. Center, Egypt, during the three summer growing seasons of 2015, 2016 and 2017. Ten yellow inbred lines of maize provided by the maize research program, Field Crop Research Institute (FCRI) of the Agricultural Research Center (ARC), Egypt, were chosen for this study. Half diallel crosses among the inbred lines was made. During 2015 growing season producing 45 yellow single crosses. The $45 \mathrm{~F}_{1}$ hybrids and two commercial hybrids as checks (single crosses 162 and 168) were evaluated in two field experiments in the years of 2016 and 2017.

The first experiment was evaluated under artificial soil inoculation by Cephalosporium maydis to study the resistance to late wilt (RLW), days to $50 \%$ silking, plant height, ear height and ear position. Meanwhile, the second experiment was done under artificial inoculation of Perenoscleroscpora sorghi to study the resistance to downy mildew (RDM). The resistant plants were taken as percentage in the two years as following.

Resistance $\%=[\underline{\text { No. of uninfected plants per plot }} \times 100]$

$$
\text { No. of total plants per plot }
$$

A randomized complete blocks design (RCBD) was used in each year for each trial with four replications. Each plot consisted of one row, $4 \mathrm{~m}$ long, $80 \mathrm{~cm}$ apart and the distance between hills was $25 \mathrm{~cm}$. All cultural practices for growing maize were conducted as recommended. Analysis of variance was carried-out according to Steel and Torrie (1980). Combined analysis was done in case of errors homogeneity Bartlett (1937). General (GCA) and specific (SCA) combining ability variance and effects were estimated according to Griffing (1956) method 4 , model 1 . The superiority percentage for different characters was measured by comparing each hybrid with the check hybrids.

\section{RESULTS AND DISCUSSION}

Analysis of variance of $45 F_{1}$ and two checks for the vegetative traits, resistance to downy mildew and late wilt diseases in two years and their combined data are presented in Table 1. Results indicated that, years (Y) mean squares were highly significant for days to $50 \%$ silking, ear height, ear position and resistance to late wilt disease, while, plant height had significant years mean squares, indicating that these traits might be changed in performance from year to another. Genotypes (G) and crosses $(\mathrm{Cr})$ mean squares were highly significant for all traits in the two years and their combined data, indicating the wide diversity between the genotypes and crosses used in this study. Checks $(\mathrm{Ch})$ mean squares were significant and / or highly significant for all traits, except for $50 \%$ silking date, resistance to downy mildew, ear height in the first year and resistance to late wilt in the first year and their combined data. $\mathrm{Cr} v s$. Ch mean squares were significant and / or highly significant for all traits, except for resistance to downy mildew in the two years and their combined data and resistance to late wilt in the second year. 


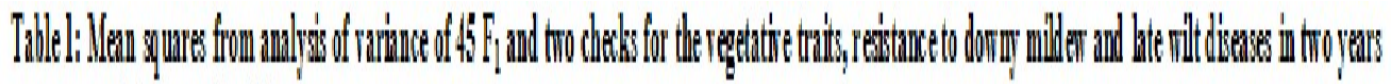

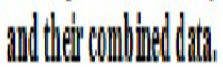

\begin{tabular}{|c|c|c|c|c|c|c|c|c|c|}
\hline \multirow[b]{2}{*}{ SOOT, } & \multicolumn{2}{|c|}{$d d^{\prime}$} & \multicolumn{2}{|c|}{ 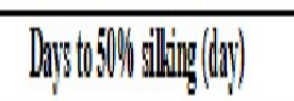 } & \multicolumn{2}{|c|}{ 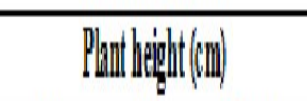 } & \multicolumn{3}{|c|}{ 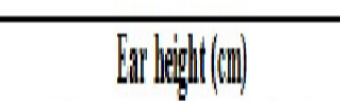 } \\
\hline & Signge & Natur & $\Pi_{1}$ & I. Cand & $\begin{array}{ll}I_{1} & I_{l}\end{array}$ & Combl. & $I_{1}$ & $I_{1}$ & Cond. \\
\hline Ferg(I) & & 1 & & 19:50\% & & $(H-1) y^{4}$ & & & (moly pla \\
\hline Ry! & & 6 & 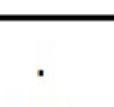 & . 6 绣 & . & 1939 & . & . & 08613 \\
\hline $\operatorname{lempge}(\theta)$ & 4 & 4 & $1966^{23}$ & 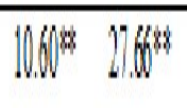 & 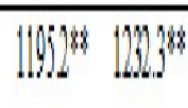 & Magk & 1.9.1.39 & Moys & 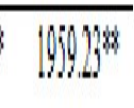 \\
\hline $\operatorname{cosex}($ (I) & H & $H$ & $17: 6^{4}$ & 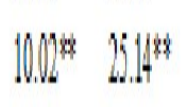 & 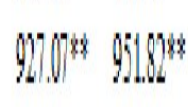 & 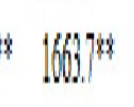 & 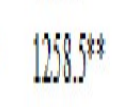 & $54.8 \%$ & $1661.13 \%$ \\
\hline 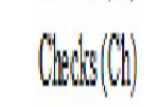 & 1 & 1 & 15iN & $11125 \quad 3.62$ & 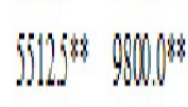 & $10066_{2}^{28}$ & $\mid W H: D$ & 121.]^{2}$ & $191.1 .60 \%$ \\
\hline Grish & 1 & 1 & 121.698 & 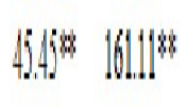 & 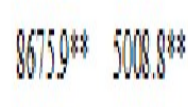 & 1394.658 & 10OA & SOM.SP & 1500.0 .30 \\
\hline Gxy & & 46 & & . $13 i 6^{*}$ & ( & 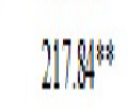 & & . & $\mid 7 j .1 .1 \%$ \\
\hline Gxy & & $H$ & & 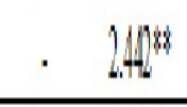 & & Uijily & & & mLj: \\
\hline Gxy! & & 1 & & . $\quad 0.663$ & . & 3610 & & $\cdot$ & 17350 \\
\hline GrabXY & . & 1 & 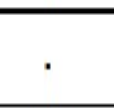 & . $9.97^{39}$ & . & 201210 & 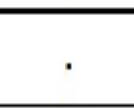 &. & $30159 y^{8}$ \\
\hline ETrO & 18 & 126 & 1.4.2 & 19951394 & 9.976 & 90601 & 86731 & 3.660 & 1000 \\
\hline
\end{tabular}

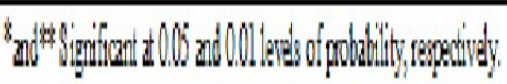




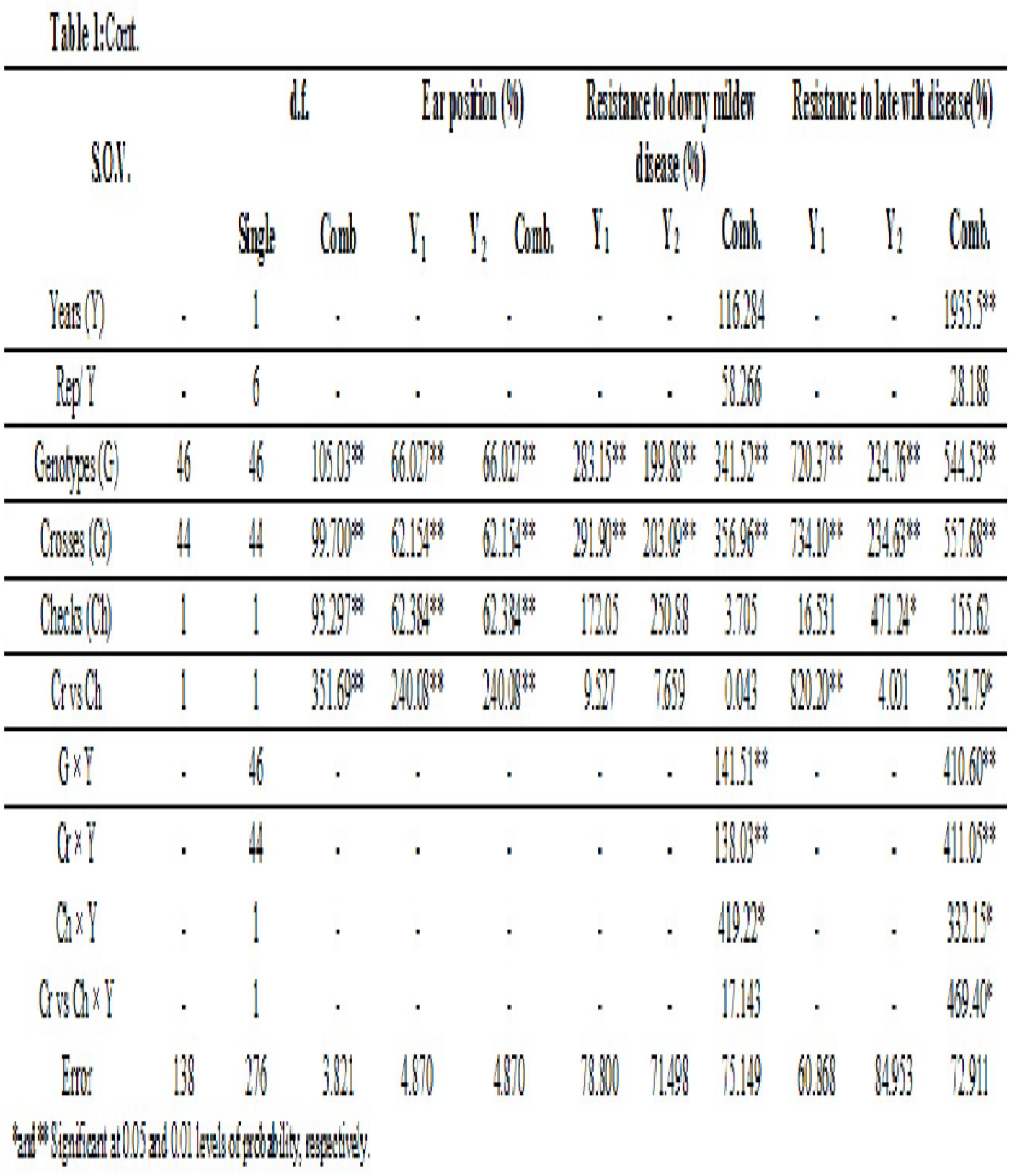


The mean squares of $\mathrm{G} \times \mathrm{Y}$ and $\mathrm{Cr} \times \mathrm{Y}$ interactions were highly significant for all traits. This results might indicate that, the performance of genotypes and crosses were affected by change in years. Ch $\mathrm{x} Y$ interaction was significant only for resistance to downy mildew and late wilt diseases. While, $\mathrm{Cr}$ vs. Ch $\mathrm{x} \mathrm{Y}$ interaction was highly significant for $50 \%$ silking date and significant only for ear height and resistance to late wilt disease. The obtained results were in good harmony with those reported by Abd El-Hadi et al. (2005) where they found that, the difference among genotypes and crosses were highly significant for days to $50 \%$ silking, plant height and ear height. While, the interaction between genotypes $\mathrm{x}$ years exhibited significant differences for days to $50 \%$ silking. Galal (2014) found that, the differences among genotypes and crosses were significant or highly significant for days to $50 \%$ silking, plant height, ear height and resistance to late wilt disease. The interaction between genotypes and crosses with environments was significant and / or highly significant for all traits. This indicated that, these genotypes and crosses differed in their order from environment to another for all traits. El-Gazzar (2015) found that, the crosses $x$ environment interaction was significant for days to $50 \%$ silking, plant height, ear height and resistance to downy mildew disease. Murtadha et al. (2016) found that, the interaction between crosses and environments was significant or highly significant for $50 \%$ silking date and plant height.

Mean performance of $45 \mathrm{~F}_{1}$ crosses and two check hybrids for the vegetative traits, resistance to downy mildew and late wilt diseases in two years and combined data are shown in Table 2. For days to 50 $\%$ silking, the crosses means ranged from 56.25 to $66.0,57.5$ to 64.75 and 56.88 to 65.25 for the first year, the second year and their combined, respectively. The crosses; $\mathrm{P}_{1} \times \mathrm{P}_{4}, \mathrm{P}_{2} \times \mathrm{P}_{3}, \mathrm{P}_{2} \times \mathrm{P}_{4}$, $\mathrm{P}_{2} \times \mathrm{P}_{5}, \mathrm{P}_{2} \times \mathrm{P}_{8}, \mathrm{P}_{2} \times \mathrm{P}_{9}, \mathrm{P}_{2} \times \mathrm{P}_{10}, \mathrm{P}_{3} \times \mathrm{P}_{8}, \mathrm{P}_{4} \times \mathrm{P}_{6}, \mathrm{P}_{4}$ $\times \mathrm{P}_{7}, \mathrm{P}_{4} \times \mathrm{P}_{8}, \mathrm{P}_{5} \times \mathrm{P}_{8}, \mathrm{P}_{6} \times \mathrm{P}_{8}, \mathrm{P}_{7} \times \mathrm{P}_{8}, \mathrm{P}_{7} \times \mathrm{P}_{9}, \mathrm{P}_{8} \times$ $\mathrm{P}_{9}, \mathrm{P}_{8} \times \mathrm{P}_{10}$ and $\mathrm{P}_{9} \times \mathrm{P}_{10}$ were significantly earlier than the earliest check SC168 in two years and their combined while, the crosses; $\mathrm{P}_{1} \times \mathrm{P}_{2}, \mathrm{P}_{1} \times \mathrm{P}_{5}, \mathrm{P}_{1} \mathrm{x}$ $\mathrm{P}_{6}, \mathrm{P}_{1} \times \mathrm{P}_{8}, \mathrm{P}_{1} \times \mathrm{P}_{9}, \mathrm{P}_{1} \times \mathrm{P}_{10}, \mathrm{P}_{2} \times \mathrm{P}_{7}, \mathrm{P}_{4} \times \mathrm{P}_{5}, \mathrm{P}_{4} \times \mathrm{P}_{9}$, $\mathrm{P}_{4} \times \mathrm{P}_{10}, \mathrm{P}_{5} \times \mathrm{P}_{7}, \mathrm{P}_{6} \times \mathrm{P}_{7}, \mathrm{P}_{6} \times \mathrm{P}_{10}$ and $\mathrm{P}_{7} \times \mathrm{P}_{10}$ were significantly earlier than both checks in the first year and their combined. Regarding plant height, the crosses means ranged from 224.25 to 290.25 , 218.75 to 279.0 and 224.88 to 284.63 for the first year, the second year and their combined, respectively. The cross $\mathrm{P}_{1} \times \mathrm{P}_{8}$ in two years and their combined had desired and significant shorter plants than both checks. While, the crosses $\mathrm{P}_{2} \times \mathrm{P}_{8}$, $\mathrm{P}_{3} \times \mathrm{P}_{8}, \mathrm{P}_{4} \times \mathrm{P}_{8}, \mathrm{P}_{7} \times \mathrm{P}_{8}, \mathrm{P}_{8} \times \mathrm{P}_{9}$ and $\mathrm{P}_{8} \times \mathrm{P}_{10}$, in the first year and their combined, the cross $\mathrm{P}_{1} \times \mathrm{P}_{3}$ in the second year and their combined and the crosses; $\mathrm{P}_{1}$ x $\mathrm{P}_{10}$ and $\mathrm{P}_{5} \times \mathrm{P}_{8}$ in the first year had desired and significant shorter plants than the shortest check SC168. Data in Table 2 concerning ear height exhibited that, the means ranged from 96.0 to 177.5 , 100.75 to 148.25 and 99.75 to 160.5 for the first year, the second year and their combined, respectively. The crosses; $\mathrm{P}_{1} \times \mathrm{P}_{2}, \mathrm{P}_{1} \times \mathrm{P}_{3}, \mathrm{P}_{1} \times \mathrm{P}_{4}$, $\mathrm{P}_{1} \times \mathrm{P}_{7}, \mathrm{P}_{1} \times \mathrm{P}_{8}, \mathrm{P}_{1} \times \mathrm{P}_{9}, \mathrm{P}_{2} \times \mathrm{P}_{7}, \mathrm{P}_{2} \times \mathrm{P}_{8}, \mathrm{P}_{2} \times \mathrm{P}_{9}, \mathrm{P}_{3}$ x $\mathrm{P}_{8}, \mathrm{P}_{4} \times \mathrm{P}_{5}, \mathrm{P}_{4} \times \mathrm{P}_{7}, \mathrm{P}_{4} \times \mathrm{P}_{8}, \mathrm{P}_{4} \times \mathrm{P}_{9}, \mathrm{P}_{5} \times \mathrm{P}_{8}, \mathrm{P}_{6} \times$ $\mathrm{P}_{7}, \mathrm{P}_{6} \times \mathrm{P}_{8}, \mathrm{P}_{7} \times \mathrm{P}_{8}, \mathrm{P}_{7} \times \mathrm{P}_{9}, \mathrm{P}_{8} \times \mathrm{P}_{9}$ and $\mathrm{P}_{8} \times \mathrm{P}_{10}$ in the two years and their combined had significant lower ear height than the two checks. While, all crosses in the first year and their combined, except $\mathrm{P}_{1} \times \mathrm{P}_{6}, \mathrm{P}_{3} \times \mathrm{P}_{4}, \mathrm{P}_{3} \times \mathrm{P}_{5}, \mathrm{P}_{3} \times \mathrm{P}_{6}, \mathrm{P}_{3} \times \mathrm{P}_{9}, \mathrm{P}_{3} \times \mathrm{P}_{10}, \mathrm{P}_{5}$ $\times \mathrm{P}_{6}, \mathrm{P}_{5} \times \mathrm{P}_{10}$ and $\mathrm{P}_{6} \times \mathrm{P}_{9}$ had significant lower ear height than the two cultivar checks. For ear position, it ranged from 42.85 to $63.46,45.05$ to 60.36 and 43.99 to 61.65 for the first year, the second year and their combined, respectively. The crosses; $\mathrm{P}_{1} \times \mathrm{P}_{2}$, $\mathrm{P}_{1} \times \mathrm{P}_{7}, \mathrm{P}_{1} \times \mathrm{P}_{8}, \mathrm{P}_{2} \times \mathrm{P}_{5}, \mathrm{P}_{2} \times \mathrm{P}_{7}, \mathrm{P}_{2} \times \mathrm{P}_{8}, \mathrm{P}_{2} \times \mathrm{P}_{9}, \mathrm{P}_{4}$ $\times \mathrm{P}_{7}, \mathrm{P}_{4} \times \mathrm{P}_{8}, \mathrm{P}_{5} \times \mathrm{P}_{7}, \mathrm{P}_{5} \times \mathrm{P}_{8}, \mathrm{P}_{6} \times \mathrm{P}_{7}, \mathrm{P}_{6} \times \mathrm{P}_{8}, \mathrm{P}_{7} \times$ $\mathrm{P}_{8}, \mathrm{P}_{7} \times \mathrm{P}_{9}$ and $\mathrm{P}_{8} \times \mathrm{P}_{9}$ in the two years and their combined and the crosses; $\mathrm{P}_{2} \times \mathrm{P}_{4}, \mathrm{P}_{2} \times \mathrm{P}_{6}, \mathrm{P}_{4} \times \mathrm{P}_{6}$ and $\mathrm{P}_{7} \times \mathrm{P}_{10}$ in the first year and their combined had significantly better ear position than the hybrid checks in comparison. Considering resistance to downy mildew disease, the means ranged from 59.25 to $100.0,58.5$ to 100.0 and 58.88 to 100.0 for the first year, the second year and their combined, respectively. The crosses; $\mathrm{P}_{1} \times \mathrm{P}_{6}, \mathrm{P}_{1} \times \mathrm{P}_{7}, \mathrm{P}_{1} \times \mathrm{P}_{10}$, $\mathrm{P}_{2} \times \mathrm{P}_{3}, \mathrm{P}_{2} \times \mathrm{P}_{4}, \mathrm{P}_{2} \times \mathrm{P}_{7}, \mathrm{P}_{3} \times \mathrm{P}_{7}, \mathrm{P}_{3} \times \mathrm{P}_{8}, \mathrm{P}_{3} \times \mathrm{P}_{9}, \mathrm{P}_{3}$ $\times \mathrm{P}_{10}, \mathrm{P}_{4} \times \mathrm{P}_{6}, \mathrm{P}_{4} \times \mathrm{P}_{7}, \mathrm{P}_{4} \times \mathrm{P}_{9}, \mathrm{P}_{5} \times \mathrm{P}_{7}, \mathrm{P}_{6} \times \mathrm{P}_{7}, \mathrm{P}_{6} \times$ $\mathrm{P}_{8}, \mathrm{P}_{6} \times \mathrm{P}_{10}, \mathrm{P}_{7} \times \mathrm{P}_{9}, \mathrm{P}_{7} \times \mathrm{P}_{10}, \mathrm{P}_{8} \times \mathrm{P}_{9}, \mathrm{P}_{8} \times \mathrm{P}_{10}$ and $\mathrm{P}_{9}$ $\mathrm{x} \mathrm{P}_{10}$ had high resistance to downy mildew disease ( more than $95 \%$ ), in two years and their combined. Regarding resistance to late wilt disease, the crosses means ranged from 44.25 to $100.0,57.5$ to 98.5 and 65.38 to 99.13 for the first year, the second year and their combined, respectively. The crosses; $\mathrm{P}_{1} \times \mathrm{P}_{4}$, $\begin{array}{llllllll}\mathrm{P}_{2} & \times & \mathrm{P}_{10} & \text { and } \mathrm{P}_{4} & \times & \mathrm{P}_{10} & \text { in both years and their }\end{array}$ combined and the crosses; $\mathrm{P}_{1} \times \mathrm{P}_{2}, \mathrm{P}_{1} \times \mathrm{P}_{3}, \mathrm{P}_{1} \times \mathrm{P}_{9}$, $\mathrm{P}_{3} \times \mathrm{P}_{5}, \mathrm{P}_{3} \times \mathrm{P}_{9}, \mathrm{P}_{3} \times \mathrm{P}_{10}, \mathrm{P}_{4} \times \mathrm{P}_{5}, \mathrm{P}_{4} \times \mathrm{P}_{6}, \mathrm{P}_{4} \times \mathrm{P}_{9}, \mathrm{P}_{7}$ $\mathrm{x} \mathrm{P}_{10}$ and $\mathrm{P}_{8} \times \mathrm{P}_{10}$ in the first year and their combined, had high resistance to late wilt disease (more than 95\%). It could be concluded from the obtained results that, the crosses; $\mathrm{P}_{1} \times \mathrm{P}_{8}, \mathrm{P}_{2} \times \mathrm{P}_{8}, \mathrm{P}_{4}$ $\times \mathrm{P}_{8}, \mathrm{P}_{7} \times \mathrm{P}_{8}, \mathrm{P}_{8} \times \mathrm{P}_{9}$ and $\mathrm{P}_{8} \times \mathrm{P}_{10}$ were better than the two hybrid checks for the vegetative traits toward earliness, shortness, low ear height and good ear position. While the crosses; $\mathrm{P}_{1} \times \mathrm{P}_{3}, \mathrm{P}_{3} \times \mathrm{P}_{5}, \mathrm{P}_{3} \times$ $\mathrm{P}_{9}, \mathrm{P}_{3} \times \mathrm{P}_{10}, \mathrm{P}_{4} \times \mathrm{P}_{5}, \mathrm{P}_{4} \times \mathrm{P}_{6}, \mathrm{P}_{4} \times \mathrm{P}_{9}, \mathrm{P}_{7} \times \mathrm{P}_{10}$ and $\mathrm{P}_{8}$ $x \mathrm{P}_{10}$ had high resistance to downy mildew and late wilt diseases (more than 95\%) over two years, indicated that these single crosses could be used in maize breeding programs for improvement of these traits. 
Table 2: Mean performance of $45 \mathrm{~F}_{1}$ crosses and two check hybrids for the vegetative traits, resistance to downy mildew and late wilt diseases in two years and combined data.

\begin{tabular}{|c|c|c|c|c|c|c|c|c|c|}
\hline \multirow[t]{2}{*}{ Crosses } & \multicolumn{3}{|c|}{$\begin{array}{l}\text { Days to 50\% silking } \\
\text { (day) }\end{array}$} & \multicolumn{3}{|c|}{ Plant height (cm) } & \multicolumn{3}{|c|}{ Ear height (cm) } \\
\hline & $Y_{1}$ & $Y_{2}$ & Comb. & $Y_{1}$ & $Y_{2}$ & Comb. & $Y_{1}$ & $Y_{2}$ & Comb. \\
\hline $\mathrm{P}_{1} \times \mathrm{P}_{2}$ & 60.25 & 62.50 & 61.38 & 249.00 & 231.75 & 240.38 & 123.25 & 111.25 & 117.25 \\
\hline$P_{1} \times P_{3}$ & 63.75 & 63.75 & 63.75 & 248.75 & 222.50 & 235.63 & 144.50 & 124.00 & 134.25 \\
\hline $\mathrm{P}_{1} \times \mathrm{P}_{4}$ & 61.75 & 62.00 & 61.88 & 260.25 & 239.00 & 249.63 & 139.25 & 127.00 & 133.13 \\
\hline $\mathrm{P}_{1} \times \mathrm{P}_{5}$ & 61.75 & 63.00 & 62.38 & 277.00 & 264.75 & 270.88 & 149.75 & 136.25 & 143.00 \\
\hline $\mathrm{P}_{1} \times \mathrm{P}_{6}$ & 61.75 & 63.25 & 62.50 & 277.50 & 254.00 & 265.75 & 154.25 & 143.25 & 148.75 \\
\hline $\mathrm{P}_{1} \times \mathrm{P}_{7}$ & 61.50 & 64.00 & 62.75 & 270.25 & 250.00 & 260.13 & 129.25 & 118.50 & 123.88 \\
\hline $\mathrm{P}_{1} \times \mathrm{P}_{8}$ & 60.00 & 62.25 & 61.13 & 231.00 & 218.75 & 224.88 & 115.75 & 109.00 & 112.38 \\
\hline $\mathrm{P}_{1} \times \mathrm{P}_{9}$ & 61.50 & 63.25 & 62.38 & 251.25 & 239.00 & 245.13 & 138.00 & 125.00 & 131.50 \\
\hline $\mathrm{P}_{1} \times \mathrm{P}_{10}$ & 61.50 & 62.75 & 62.13 & 246.25 & 241.75 & 244.00 & 140.75 & 135.75 & 138.25 \\
\hline $\mathrm{P}_{2} \times \mathrm{P}_{3}$ & 60.75 & 61.50 & 61.13 & 256.00 & 242.00 & 249.00 & 144.50 & 130.50 & 137.50 \\
\hline $\mathrm{P}_{2} \times \mathrm{P}_{4}$ & 59.00 & 61.75 & 60.38 & 273.25 & 268.50 & 270.88 & 142.00 & 136.25 & 139.13 \\
\hline $\mathrm{P}_{2} \times \mathrm{P}_{5}$ & 58.00 & 61.50 & 59.75 & 267.25 & 265.00 & 266.13 & 132.00 & 132.25 & 132.13 \\
\hline $\mathrm{P}_{2} \times \mathrm{P}_{6}$ & 62.25 & 63.75 & 63.00 & 267.75 & 267.50 & 267.63 & 134.75 & 135.00 & 134.88 \\
\hline $\mathrm{P}_{2} \times \mathrm{P}_{7}$ & 61.00 & 63.50 & 62.25 & 255.50 & 246.00 & 250.75 & 111.00 & 113.50 & 112.25 \\
\hline $\mathrm{P}_{2} \times \mathrm{P}_{8}$ & 56.25 & 57.50 & 56.88 & 224.25 & 229.50 & 226.88 & 96.00 & 103.50 & 99.75 \\
\hline $\mathrm{P}_{2} \times \mathrm{P}_{9}$ & 59.00 & 61.00 & 60.00 & 261.00 & 261.00 & 261.00 & 123.00 & 119.75 & 121.38 \\
\hline $\mathrm{P}_{2} \times \mathrm{P}_{10}$ & 59.00 & 61.00 & 60.00 & 269.25 & 257.25 & 263.25 & 142.00 & 138.75 & 140.38 \\
\hline $\mathrm{P}_{3} \times \mathrm{P}_{4}$ & 62.25 & 63.00 & 62.63 & 260.75 & 230.25 & 245.50 & 163.00 & 139.00 & 151.00 \\
\hline $\mathrm{P}_{3} \times \mathrm{P}_{5}$ & 62.50 & 63.00 & 62.75 & 273.75 & 244.50 & 259.13 & 167.00 & 138.50 & 152.75 \\
\hline $\mathrm{P}_{3} \times \mathrm{P}_{6}$ & 66.00 & 64.50 & 65.25 & 281.25 & 248.75 & 265.00 & 177.50 & 143.50 & 160.50 \\
\hline $\mathrm{P}_{3} \times \mathrm{P}_{7}$ & 62.00 & 62.50 & 62.25 & 259.75 & 246.00 & 252.88 & 141.50 & 136.50 & 139.00 \\
\hline $\mathrm{P}_{3} \times \mathrm{P}_{8}$ & 59.25 & 60.75 & 60.00 & 244.25 & 222.75 & 233.50 & 134.25 & 120.00 & 127.13 \\
\hline $\mathrm{P}_{3} \times \mathrm{P}_{9}$ & 64.00 & 63.75 & 63.88 & 277.25 & 241.25 & 259.25 & 169.00 & 133.50 & 151.25 \\
\hline $\mathrm{P}_{3} \times \mathrm{P}_{10}$ & 62.75 & 64.75 & 63.75 & 257.50 & 230.25 & 243.88 & 163.50 & 137.75 & 150.63 \\
\hline $\mathrm{P}_{4} \times \mathrm{P}_{5}$ & 59.00 & 62.25 & 60.63 & 251.00 & 241.00 & 246.00 & 135.50 & 129.50 & 132.50 \\
\hline $\mathrm{P}_{4} \times \mathrm{P}_{6}$ & 59.75 & 61.50 & 60.63 & 265.00 & 248.25 & 256.63 & 136.25 & 129.75 & 133.00 \\
\hline $\mathrm{P}_{4} \times \mathrm{P}_{7}$ & 60.75 & 62.00 & 61.38 & 256.00 & 248.25 & 252.13 & 123.50 & 119.00 & 121.25 \\
\hline $\mathrm{P}_{4} \times \mathrm{P}_{8}$ & 57.00 & 60.00 & 58.50 & 239.75 & 226.50 & 233.13 & 117.00 & 112.50 & 114.75 \\
\hline $\mathrm{P}_{4} \times \mathrm{P}_{9}$ & 61.25 & 62.75 & 62.00 & 252.75 & 236.75 & 244.75 & 133.75 & 118.25 & 126.00 \\
\hline $\mathrm{P}_{4} \times \mathrm{P}_{10}$ & 61.50 & 63.00 & 62.25 & 250.00 & 247.50 & 248.75 & 144.00 & 135.25 & 139.63 \\
\hline $\mathrm{P}_{5} \times \mathrm{P}_{6}$ & 63.25 & 63.00 & 63.13 & 279.75 & 265.00 & 272.38 & 154.75 & 136.75 & 145.75 \\
\hline $\mathrm{P}_{5} \times \mathrm{P}_{7}$ & 61.75 & 63.25 & 62.50 & 290.25 & 279.00 & 284.63 & 149.00 & 136.00 & 142.50 \\
\hline $\mathrm{P}_{5} \times \mathrm{P}_{8}$ & 57.25 & 59.75 & 58.50 & 242.00 & 243.00 & 242.50 & 114.50 & 115.50 & 115.00 \\
\hline $\mathrm{P}_{5} \times \mathrm{P}_{9}$ & 62.25 & 64.50 & 63.38 & 278.00 & 263.50 & 270.75 & 150.50 & 138.00 & 144.25 \\
\hline $\mathrm{P}_{5} \times \mathrm{P}_{10}$ & 62.00 & 63.50 & 62.75 & 274.25 & 265.25 & 269.75 & 156.50 & 148.25 & 152.38 \\
\hline $\mathrm{P}_{6} \times \mathrm{P}_{7}$ & 60.50 & 62.25 & 61.38 & 262.00 & 262.50 & 262.25 & 125.25 & 121.00 & 123.13 \\
\hline $\mathrm{P}_{6} \times \mathrm{P}_{8}$ & 59.00 & 59.25 & 59.13 & 250.25 & 233.25 & 241.75 & 125.50 & 112.25 & 118.88 \\
\hline $\mathrm{P}_{6} \times \mathrm{P}_{9}$ & 63.00 & 63.50 & 63.25 & 270.50 & 267.75 & 269.13 & 152.50 & 145.75 & 149.13 \\
\hline $\mathrm{P}_{6} \times \mathrm{P}_{10}$ & 59.75 & 62.50 & 61.13 & 261.50 & 253.75 & 257.63 & 145.75 & 136.00 & 140.88 \\
\hline $\mathrm{P}_{7} \mathrm{x}_{\mathrm{P}}$ & 57.75 & 60.50 & 59.13 & 230.25 & 229.00 & 229.63 & 102.75 & 108.00 & 105.38 \\
\hline $\mathrm{P}_{7} \times \mathrm{P}_{9}$ & 61.25 & 62.00 & 61.63 & 257.25 & 266.75 & 262.00 & 128.00 & 123.00 & 125.50 \\
\hline $\mathrm{P}_{7} \times \mathrm{P}_{10}$ & 60.75 & 62.25 & 61.50 & 268.00 & 262.75 & 265.38 & 137.50 & 133.75 & 135.63 \\
\hline $\mathrm{P}_{8} \mathrm{XP}_{9}$ & 57.50 & 58.25 & 57.88 & 226.75 & 223.75 & 225.25 & 106.75 & 100.75 & 103.75 \\
\hline $\mathrm{P}_{8} \times \mathrm{P}_{10}$ & 56.75 & 60.50 & 58.63 & 246.00 & 230.75 & 238.38 & 132.50 & 116.75 & 124.63 \\
\hline $\mathrm{P}_{9} \times \mathrm{P}_{10}$ & 61.75 & 61.50 & 61.63 & 256.75 & 255.50 & 256.13 & 140.00 & 135.75 & 137.88 \\
\hline Checks & 65.50 & 65.00 & 65.25 & 318.75 & 307.50 & 313.13 & 179.75 & 167.50 & 173.63 \\
\hline $\begin{array}{ll}\text { SC168 } \\
\end{array}$ & 64.00 & 64.25 & 64.13 & 266.25 & 237.50 & 251.88 & 168.25 & 142.75 & 155.50 \\
\hline & 2.19 & .07 & 1.51 & 17.63 & 14.80 & 11.51 & 16.98 & 13.49 & 10.84 \\
\hline
\end{tabular}


Table 2: Cont.

\begin{tabular}{|c|c|c|c|c|c|c|c|c|c|}
\hline \multirow[t]{2}{*}{ Crosses } & \multicolumn{3}{|c|}{ Ear position (\%) } & \multicolumn{3}{|c|}{$\begin{array}{l}\text { Resistance to downy } \\
\text { mildew disease }(\%)\end{array}$} & \multicolumn{3}{|c|}{$\begin{array}{l}\text { Resistance to late wilt } \\
\text { disease }(\%)\end{array}$} \\
\hline & $\mathbf{Y}_{1}$ & $\mathbf{Y}_{2}$ & Comb. & $\mathrm{Y}_{1}$ & $\mathbf{Y}_{2}$ & Comb. & $\mathrm{Y}_{1}$ & $\mathbf{Y}_{2}$ & Comb. \\
\hline $\mathrm{P}_{1} \times \mathrm{P}_{2}$ & 49.53 & 48.08 & 48.80 & 88.75 & 98.25 & 93.50 & 100.00 & 90.50 & 95.25 \\
\hline $\mathrm{P}_{1} \times \mathrm{P}_{3}$ & 58.06 & 55.71 & 56.88 & 92.00 & 100.00 & 96.00 & 99.00 & 91.50 & 95.25 \\
\hline $\mathrm{P}_{1} \times \mathrm{P}_{4}$ & 53.50 & 53.05 & 53.28 & 89.00 & 91.75 & 90.38 & 100.00 & 97.50 & 98.75 \\
\hline $\mathrm{P}_{1} \times \mathrm{P}_{5}$ & 54.13 & 51.48 & 52.81 & 100.00 & 94.75 & 97.38 & 58.00 & 95.25 & 76.63 \\
\hline $\mathrm{P}_{1} \times \mathrm{P}_{6}$ & 55.67 & 56.38 & 56.02 & 100.00 & 95.25 & 97.63 & 87.00 & 95.75 & 91.38 \\
\hline $\mathrm{P}_{1} \times \mathrm{P}_{7}$ & 47.89 & 47.50 & 47.69 & 97.00 & 97.50 & 97.25 & 100.00 & 86.50 & 93.25 \\
\hline $\mathrm{P}_{1} \times \mathrm{P}_{8}$ & 50.10 & 49.83 & 49.96 & 94.50 & 97.00 & 95.75 & 68.25 & 76.25 & 72.25 \\
\hline $\mathrm{P}_{1} \times \mathrm{P}_{9}$ & 54.97 & 52.30 & 53.64 & 85.75 & 86.75 & 86.25 & 100.00 & 93.00 & 96.50 \\
\hline $\mathrm{P}_{1} \times \mathrm{P}_{10}$ & 57.13 & 56.12 & 56.62 & 98.50 & 97.25 & 97.88 & 98.00 & 85.50 & 91.75 \\
\hline $\mathrm{P}_{2} \times \mathrm{P}_{3}$ & 56.42 & 53.96 & 55.19 & 97.50 & 100.00 & 98.75 & 100.00 & 88.50 & 94.25 \\
\hline $\mathrm{P}_{2} \times \mathrm{P}_{4}$ & 51.95 & 50.72 & 51.34 & 100.00 & 98.50 & 99.25 & 83.50 & 90.75 & 87.13 \\
\hline $\mathrm{P}_{2} \times \mathrm{P}_{5}$ & 49.39 & 50.00 & 49.69 & 93.25 & 94.00 & 93.63 & 96.00 & 89.25 & 92.63 \\
\hline $\mathrm{P}_{2} \times \mathrm{P}_{6}$ & 50.27 & 50.46 & 50.36 & 95.00 & 97.25 & 96.13 & 87.00 & 93.00 & 90.00 \\
\hline $\mathrm{P}_{2} \times \mathrm{P}_{7}$ & 43.46 & 46.12 & 44.79 & 97.75 & 98.75 & 98.25 & 95.25 & 87.25 & 91.25 \\
\hline $\mathrm{P}_{2} \times \mathrm{P}_{8}$ & 42.85 & 45.12 & 43.99 & 81.75 & 98.50 & 90.13 & 48.00 & 82.75 & 65.38 \\
\hline $\mathrm{P}_{2} \times \mathrm{P}_{9}$ & 47.13 & 45.86 & 46.49 & 59.25 & 58.50 & 58.88 & 94.25 & 95.00 & 94.63 \\
\hline $\mathrm{P}_{2} \times \mathrm{P}_{10}$ & 52.71 & 53.90 & 53.31 & 88.00 & 93.50 & 90.75 & 99.00 & 98.50 & 98.75 \\
\hline $\mathrm{P}_{3} \times \mathrm{P}_{4}$ & 62.46 & 60.36 & 61.41 & 83.75 & 98.50 & 91.13 & 100.00 & 90.00 & 95.00 \\
\hline $\mathrm{P}_{3} \times \mathrm{P}_{5}$ & 61.01 & 56.64 & 58.83 & 93.75 & 100.00 & 96.88 & 97.00 & 94.25 & 95.63 \\
\hline $\mathrm{P}_{3} \times \mathrm{P}_{6}$ & 63.11 & 57.55 & 60.33 & 93.25 & 98.50 & 95.88 & 100.00 & 86.00 & 93.00 \\
\hline $\mathrm{P}_{3} \times \mathrm{P}_{7}$ & 54.43 & 55.45 & 54.94 & 97.75 & 100.00 & 98.88 & 97.00 & 92.00 & 94.50 \\
\hline $\mathrm{P}_{3} \times \mathrm{P}_{8}$ & 54.97 & 53.87 & 54.42 & 100.00 & 96.50 & 98.25 & 100.00 & 89.00 & 94.50 \\
\hline $\mathrm{P}_{3} \times \mathrm{P}_{9}$ & 60.95 & 55.19 & 58.07 & 98.00 & 96.00 & 97.00 & 100.00 & 92.00 & 96.00 \\
\hline $\mathrm{P}_{3} \times \mathrm{P}_{10}$ & 63.46 & 59.85 & 61.65 & 97.50 & 100.00 & 98.75 & 100.00 & 94.00 & 97.00 \\
\hline $\mathrm{P}_{4} \times \mathrm{P}_{5}$ & 53.98 & 53.71 & 53.85 & 100.00 & 94.00 & 97.00 & 97.50 & 93.50 & 95.50 \\
\hline $\mathrm{P}_{4} \times \mathrm{P}_{6}$ & 51.35 & 52.27 & 51.81 & 100.00 & 98.75 & 99.38 & 100.00 & 90.00 & 95.00 \\
\hline $\mathrm{P}_{4} \times \mathrm{P}_{7}$ & 48.30 & 47.90 & 48.10 & 97.00 & 98.00 & 97.50 & 90.75 & 88.75 & 89.75 \\
\hline $\mathrm{P}_{4} \times \mathrm{P}_{8}$ & 48.85 & 49.66 & 49.25 & 100.00 & 91.50 & 95.75 & 96.75 & 90.25 & 93.50 \\
\hline $\mathrm{P}_{4} \times \mathrm{P}_{9}$ & 52.94 & 49.96 & 51.45 & 100.00 & 97.50 & 98.75 & 100.00 & 91.25 & 95.63 \\
\hline $\mathrm{P}_{4} \times \mathrm{P}_{10}$ & 57.54 & 54.63 & 56.08 & 97.00 & 92.50 & 94.75 & 100.00 & 98.25 & 99.13 \\
\hline $\mathrm{P}_{5} \times \mathrm{P}_{6}$ & 55.32 & 51.57 & 53.44 & 100.00 & 90.25 & 95.13 & 82.00 & 97.00 & 89.50 \\
\hline $\mathrm{P}_{5} \times \mathrm{P}_{7}$ & 51.38 & 48.71 & 50.04 & 96.00 & 98.75 & 97.38 & 86.00 & 91.75 & 88.88 \\
\hline $\mathrm{P}_{5} \times \mathrm{P}_{8}$ & 47.28 & 47.56 & 47.42 & 94.75 & 90.75 & 92.75 & 67.75 & 89.75 & 78.75 \\
\hline $\mathrm{P}_{5} \times \mathrm{P}_{9}$ & 54.09 & 52.36 & 53.23 & 88.25 & 99.00 & 93.63 & 82.25 & 89.50 & 85.88 \\
\hline $\mathrm{P}_{5} \times \mathrm{P}_{10}$ & 57.07 & 55.87 & 56.47 & 84.50 & 98.50 & 91.50 & 96.75 & 92.50 & 94.63 \\
\hline $\mathrm{P}_{6} \times \mathrm{P}_{7}$ & 47.63 & 45.97 & 46.80 & 96.75 & 99.00 & 97.88 & 44.25 & 97.25 & 70.75 \\
\hline $\mathrm{P}_{6} \times \mathrm{P}_{8}$ & 50.19 & 48.12 & 49.16 & 97.25 & 98.75 & 98.00 & 95.50 & 80.75 & 88.13 \\
\hline $\mathrm{P}_{6} \times \mathrm{P}_{9}$ & 56.33 & 54.44 & 55.38 & 100.00 & 92.50 & 96.25 & 76.25 & 82.25 & 79.25 \\
\hline $\mathrm{P}_{6} \times \mathrm{P}_{10}$ & 55.66 & 53.59 & 54.62 & 100.00 & 100.00 & 100.00 & 91.75 & 96.75 & 94.25 \\
\hline $\mathrm{P}_{7} \mathrm{x} \mathrm{P}_{8}$ & 44.61 & 47.19 & 45.90 & 100.00 & 90.00 & 95.00 & 78.75 & 57.50 & 68.13 \\
\hline $\mathrm{P}_{7} \times \mathrm{P}_{9}$ & 49.74 & 46.12 & 47.93 & 100.00 & 99.00 & 99.50 & 85.75 & 76.75 & 81.25 \\
\hline $\mathrm{P}_{7} \times \mathrm{P}_{10}$ & 51.33 & 50.97 & 51.15 & 98.50 & 100.00 & 99.25 & 99.00 & 94.25 & 96.63 \\
\hline $\mathrm{P}_{8} \mathrm{X} \mathrm{P}_{9}$ & 47.12 & 45.05 & 46.08 & 99.00 & 99.00 & 99.00 & 91.75 & 71.75 & 81.75 \\
\hline $\mathrm{P}_{8} \times \mathrm{P}_{10}$ & 53.87 & 50.66 & 52.26 & 98.25 & 100.00 & 99.13 & 98.75 & 92.75 & 95.75 \\
\hline $\mathrm{P}_{9} \times \mathrm{P}_{10}$ & 54.54 & 53.13 & 53.83 & 98.00 & 99.00 & 98.50 & 99.00 & 90.50 & 94.75 \\
\hline SC162 & 56.35 & 54.47 & 55.41 & 89.25 & 100.00 & 94.63 & 99.00 & 95.25 & 97.13 \\
\hline SC168 & 63.18 & 60.06 & 61.62 & 98.00 & 95.00 & 96.50 & 100.00 & 82.50 & 91.25 \\
\hline LSD & 3.56 & 4.02 & 2.68 & 16.19 & 15.42 & 11.18 & 14.23 & 16.81 & 11.01 \\
\hline
\end{tabular}


General and specific combining ability mean squares and their interactions with years for the vegetative traits, resistance to downy mildew and late wilt diseases in two years and their combined data are shown in Table 3. The results showed that, mean squares due to general combining ability (GCA) and specific combining ability (SCA) were highly significant for all traits in two years and their combined, indicating that additive and non-additive gene action had an important role in the inheritance of all traits. On the other side, the interactions between GCA and SCA with years were highly significant for all traits, indicating that both types of gene action were influenced by change of years. Regarding GCA / SCA mean square ratio, the data pointed out that, this ratio exceeded the unity for all studied traits in two years and their combined. This might indicates that, additive and additive $\mathrm{x}$ additive gene effects were more important than non-additive gene effects for the inheritance of all studied traits. The ratio of GCA x Y / SCA x Y mean square found to be exceeded the unity for all traits, indicating that the additive gene effects were more interacted with years than non-additive ones for all traits. These results agreed with those obtained by Abd El-Hadi et al. (2005) which reported that, the mean squares of GCA and SCA were highly significant for all studied traits i.e. ; days to $50 \%$ silking, plant height and ear height. The ratio GCA/SCA mean squares exceeded the unity for plant height, while the ratio GCA $x$ Y/ SCA x Y mean squares exceeded the unity for days to $50 \%$ silking.

Motawei (2006) found that, highly significant mean squares due to GCA and SCA were detected for days to $50 \%$ silking, plant height and resistance to late wilt, indicating that both additive and nonadditive gene effects are operating in the inheritance of these traits. However, additive gene effects exhibited larger contribution in the genetic control of these traits than those of non-additive genetic effects, since the ratio GCA/SCA mean squares exceeded the unity. Akanda et al. (2007) found that, mean squares due to GCA and SCA were significant for days to $50 \%$ silking, plant and ear height. However, additive gene effects were preponderance for the expression for these traits. Moradi (2014) found that, GCA and SCA were significant for days to $50 \%$ silking and plant height, indicating that additive and non-additive gene action had an important role in the inheritance of these traits. The GCA x year interaction was significant for plant height. El-Gazzar (2015) found that, GCA/SCA ratios revealed that the additive types of gene action were the most important expressions for days to $50 \%$ silking, plant height, ear height and resistance to downy mildew disease. El-Shamarka et al. (2015) found that, mean squares due to GCA and SCA were significant for number of days to $50 \%$ silking, plant height and ear height. The ratio of GCA / SCA mean square exceeded the unity for all traits, while GCA $\mathrm{x}$ environment / SCA $\mathrm{x}$ environment mean square exceeded the unity for all traits, except days to $50 \%$ silking.

Estimates of general combining ability effects ( $\left.\mathbf{g}^{\wedge} \mathbf{i}\right)$ for the vegetative traits, resistance to downy mildew and late wilt diseases in two years and their combined data are presented in Table 4 . Negative values of general combining ability effects would be desirable from the breeder point of view for all traits, except for resistance to downy mildew and late wilt diseases, where the positive values would be favored. General combining ability effects $\left(\mathbf{g}^{\wedge} \mathbf{i}\right)$ would be estimated wherever the significant of GCA mean square for the trait in view. The $\left(\mathbf{g}_{\mathbf{i}} \mathbf{i}\right)$ would be considered, when their GCA mean squares is not significant. The parental inbred line; $\mathrm{P}_{2}$ might be considered as a good combiner for days to $50 \%$ silking, ear height and ear position in the two years and their combined data due to its negative and highly significant $\left(\mathbf{g}_{\mathbf{i}}\right)$ values in all cases. The parental inbred line; $\mathrm{P}_{8}$ had highly significant $\left(\mathbf{g}^{\wedge} \mathbf{i}\right)$ values in negative direction for days to $50 \%$ silking, plant height, ear height and ear position in two years and their combined analysis. That parent might be considered as a good combiner for the traits in view in the two studied years. The parental inbred line; $\mathrm{P}_{7}$ had highly significant $\left(\mathbf{g}_{\mathbf{i}} \mathbf{i}\right)$ values in negative direction for ear height and ear position in both years, which might considers as a good combiner parent for the traits in question, and the parental inbred lines; $\mathrm{P}_{3}$ and $\mathrm{P}_{4}$ considered as good combiner for plant height in the second year due to its negative and significant $\left(\mathbf{g}^{\wedge} \mathbf{i}\right)$ values in this concern. The parental inbred line; $\mathrm{P}_{7}$ in the two years and $\mathrm{P}_{6}$ in the first year had significant $\left(\mathbf{g}^{\wedge} \mathbf{i}\right)$ values in positive direction for resistance to downy mildew disease. While, the parental inbred line; $\mathrm{P}_{4}$ and $\mathrm{P}_{10}$ in the two years and $\mathrm{P}_{3}$ in the first year had significant and positive $\left(\mathbf{g}^{\wedge} \mathbf{i}\right)$ values for the resistance to late wilt disease. These parental inbred line might be considered a good combiner for resistance to the diseases. From the obtained results, it could be summarized that, the inbred line $\mathrm{P}_{8}$ had desirable significant of $\left(\mathbf{g}_{\mathbf{i}} \mathbf{i}\right)$ for earliness and dwarfnees, which might indicated that this inbred line considered a good combiner for to generating genotype characterized by earliness and shortness. The inbred line $\mathrm{P}_{7}$ for resistance to downy mildew and suitable ear height ( position ) and, also the inbred line $\mathrm{P}_{4}$ for earliness, shortness and resistance to late wilt disease. 
Table 3:General and specific combining ability mean squares and their interactions with years for the vegetative traits, resistance to downy mildew and late wilt diseases in two years and their combined data.

\begin{tabular}{|c|c|c|c|c|c|c|c|c|c|c|c|}
\hline \multirow{2}{*}{ S.O.V. } & \multicolumn{2}{|c|}{ d.f. } & \multicolumn{3}{|c|}{$\begin{array}{c}\begin{array}{c}\text { Days to } 50 \% \text { silking } \\
\text { (day) }\end{array} \\
\end{array}$} & \multicolumn{3}{|c|}{ Plant height (cm) } & \multicolumn{3}{|c|}{ Ear height (cm) } \\
\hline & Single & Comb. & $\mathbf{Y}_{1}$ & $\mathbf{Y}_{2}$ & Comb. & $\mathbf{Y}_{1}$ & $\mathbf{Y}_{2}$ & Comb. & $\mathbf{Y}_{1}$ & $\mathbf{Y}_{2}$ & Comb. \\
\hline $\mathrm{GCA}$ & 9 & 9 & $17.210^{* *}$ & $8.698^{* *}$ & $12.260^{* *}$ & $809.261^{* *}$ & $936.287^{* *}$ & $786.338^{* *}$ & $1322.34 * *$ & $556.078^{* *}$ & $864.677^{* *}$ \\
\hline $\mathrm{SCA}$ & 35 & 35 & $1.095 * *$ & $0.914 * *$ & $0.799 * *$ & $83.270^{* *}$ & $58.386^{* *}$ & $59.252 * *$ & $55.504 * *$ & $37.655^{* *}$ & $38.689^{* *}$ \\
\hline $\begin{array}{c}\mathrm{GCA} \times \\
\mathrm{Y} \\
\end{array}$ & - & 9 & - & - & $13.648 * *$ & - & - & $959.210^{* *}$ & - & - & $1013.74^{* *}$ \\
\hline $\begin{array}{c}\mathrm{SCA} \times \\
\mathrm{Y}\end{array}$ & - & 35 & - & - & $1.210^{* *}$ & - & - & $82.404 * *$ & - & - & $54.470^{* *}$ \\
\hline $\begin{array}{l}\text { Error } \\
\text { term }\end{array}$ & 132 & 264 & 0.368 & 0.325 & 0.346 & 23.237 & 16.280 & 19.758 & 20.912 & 13.646 & 17.279 \\
\hline $\begin{array}{c}\mathrm{GCA} / \\
\mathrm{SCA} \\
\end{array}$ & - & - & 15.716 & 9.516 & 15.344 & 9.718 & 16.036 & 13.271 & 23.824 & 14.767 & 22.349 \\
\hline $\begin{array}{c}\mathrm{GCA} \times \\
\mathrm{Y} / \\
\mathrm{SCA} \times \\
\mathrm{Y}\end{array}$ & - & - & - & - & 11.279 & - & - & 11.640 & - & - & 18.610 \\
\hline
\end{tabular}

Table3:Cont.

\begin{tabular}{|c|c|c|c|c|c|c|c|c|c|c|c|}
\hline \multirow{2}{*}{ S.O.V. } & \multicolumn{2}{|c|}{ d.f. } & \multicolumn{3}{|c|}{ Ear position (\%) } & \multicolumn{3}{|c|}{$\begin{array}{l}\text { Resistance to downy } \\
\text { mildew disease }(\%)\end{array}$} & \multicolumn{3}{|c|}{$\begin{array}{c}\text { Resistance to late wilt } \\
\text { disease }(\%)\end{array}$} \\
\hline & Single & Comb. & $Y_{1}$ & $\mathbf{Y}_{2}$ & Comb. & $Y_{1}$ & $\mathbf{Y}_{2}$ & Comb. & $Y_{1}$ & $\mathbf{Y}_{2}$ & Comb. \\
\hline GCA & 9 & 9 & $113.075^{* *}$ & $66.019^{* *}$ & $87.104^{* *}$ & $106.834^{* *}$ & $63.094 * *$ & $64.389^{* *}$ & $409.663^{* *}$ & $139.861^{* *}$ & $191.948^{* *}$ \\
\hline SCA & 35 & 35 & $2.258^{* *}$ & $2.558^{* * *}$ & $1.846^{* *}$ & $64.269 * *$ & $47.606 * *$ & $39.537^{* *}$ & $125.376^{* *}$ & $37.779 * *$ & $38.279 * *$ \\
\hline $\begin{array}{c}\mathrm{GCA} \times \\
\mathrm{Y}\end{array}$ & - & 9 & - & - & $91.990 * *$ & - & - & $105.535^{* *}$ & - & - & $357.576^{* *}$ \\
\hline $\begin{array}{c}\mathrm{SCA} \times \\
\mathrm{Y}\end{array}$ & - & 35 & - & - & $2.970^{* *}$ & - & - & $72.338 * *$ & - & - & $124.876^{* *}$ \\
\hline $\begin{array}{l}\text { Error } \\
\text { term }\end{array}$ & 132 & 264 & 0.928 & 1.239 & 1.083 & 18.753 & 18.366 & 18.559 & 15.726 & 21.399 & 18.562 \\
\hline $\begin{array}{c}\mathrm{GCA} / \\
\mathrm{SCA}\end{array}$ & - & - & 50.077 & 25.808 & 47.185 & 1.662 & 1.325 & 1.628 & 3.267 & 3.702 & 5.014 \\
\hline $\begin{array}{c}\mathrm{GCA} \times \\
\mathrm{Y} / \\
\mathrm{SCA} \times \\
\mathrm{Y}\end{array}$ & - & - & - & - & 30.973 & - & - & 1.458 & - & - & 2.863 \\
\hline
\end{tabular}


Table 4:Estimates of general combining ability effects $\left(g^{\wedge}\right)$ for the vegetative traits, resistance to downy mildew and late wilt diseases in two years and their combined data.

\begin{tabular}{|c|c|c|c|c|c|c|c|c|c|c|}
\hline \multirow{2}{*}{\multicolumn{2}{|c|}{$\begin{array}{c}\text { Inbred } \\
\text { lines }\end{array}$}} & \multicolumn{3}{|c|}{ Days to $50 \%$ silking (day) } & \multicolumn{3}{|c|}{ Plant height (cm) } & \multicolumn{3}{|c|}{ Ear height (cm) } \\
\hline & & $Y_{1}$ & $\mathbf{Y}_{2}$ & Comb. & $Y_{1}$ & $\mathbf{Y}_{2}$ & Comb. & $Y_{1}$ & $\mathbf{Y}_{2}$ & Comb. \\
\hline \multicolumn{2}{|c|}{$\mathrm{P}_{1}$} & $0.931 * *$ & $0.881 * *$ & $0.906^{* *}$ & -2.294 & $-7.606 * *$ & $-4.950 * *$ & -0.344 & -2.250 & -1.297 \\
\hline \multicolumn{2}{|c|}{$\mathrm{P}_{2}$} & $-1.350 * *$ & $-0.713^{* *}$ & $-1.031 * *$ & -0.794 & $5.769 * *$ & 2.488 & $-11.125^{* *}$ & $-3.406^{* *}$ & $-7.266^{* *}$ \\
\hline \multicolumn{2}{|c|}{$\mathrm{P}_{3}$} & $2.119 * *$ & $0.975 * *$ & $1.547 * *$ & $3.706^{*}$ & $-11.763^{* *}$ & $-4.028 * *$ & $20.906^{* *}$ & $6.906 * *$ & $13.906^{* *}$ \\
\hline \multicolumn{2}{|c|}{$\mathrm{P}_{4}$} & $-0.506^{*}$ & -0.181 & -0.344 & -2.606 & $-4.544 * *$ & $-3.575^{*}$ & -0.406 & -0.188 & -0.297 \\
\hline \multicolumn{2}{|c|}{$\mathrm{P}_{5}$} & 0.181 & $0.506^{* *}$ & 0.344 & $12.956^{* *}$ & $13.581 * *$ & $13.269^{* *}$ & $9.000 * *$ & $7.875^{* *}$ & $8.438 * *$ \\
\hline \multicolumn{2}{|c|}{$\mathrm{P}_{6}$} & $1.119 * *$ & $0.475^{*}$ & $0.797 * *$ & $10.738 * *$ & $9.800 * *$ & $10.269^{* *}$ & $8.625 * *$ & $6.906 * *$ & $7.766^{* *}$ \\
\hline \multicolumn{2}{|c|}{$\mathrm{P}_{7}$} & 0.119 & 0.319 & 0.219 & 2.456 & $8.488^{* *}$ & $5.472 * *$ & $-11.219^{* *}$ & $-4.844 * *$ & $-8.031 * *$ \\
\hline \multicolumn{2}{|c|}{$\mathrm{P}_{8}$} & $-3.194 * *$ & $-2.619^{* *}$ & $-2.906^{* *}$ & $-24.388 * *$ & $-20.638^{* *}$ & $-22.513^{* *}$ & $-24.063^{* *}$ & $-18.719^{* *}$ & $-21.391 * *$ \\
\hline \multicolumn{2}{|c|}{$\mathrm{P}_{9}$} & $0.650 * *$ & 0.100 & 0.375 & 0.238 & $4.113 * *$ & 2.175 & 0.500 & -1.031 & -0.266 \\
\hline \multicolumn{2}{|c|}{$\mathrm{P}_{10}$} & -0.069 & 0.256 & 0.094 & -0.013 & $2.800 *$ & 1.394 & $8.125 * *$ & 8.750 ** & $8.438 * *$ \\
\hline \multirow{2}{*}{$\begin{array}{l}\text { L.S.D } \\
\text { gi }\end{array}$} & 0.05 & 0.399 & 0.375 & 0.387 & 3.169 & 2.653 & 2.911 & 3.006 & 2.428 & 2.717 \\
\hline & 0.01 & 0.525 & 0.494 & 0.510 & 4.171 & 3.492 & 3.832 & 3.957 & 3.197 & 3.577 \\
\hline \multirow{2}{*}{$\begin{array}{l}\text { L.S.D } \\
\text { gi-gj }\end{array}$} & 0.05 & 0.595 & 0.559 & 0.577 & 4.724 & 3.954 & 4.339 & 4.482 & 3.620 & 4.051 \\
\hline & 0.01 & 0.783 & 0.736 & 0.760 & 6.218 & 5.205 & 5.712 & 5.899 & 4.765 & 5.332 \\
\hline
\end{tabular}

*and** Significant at 0.05 and 0.01 levels of probability, respectively.

Table 4: Cont.

\begin{tabular}{|c|c|c|c|c|c|c|c|c|c|c|}
\hline \multirow{2}{*}{\multicolumn{2}{|c|}{ Inbred lines }} & \multicolumn{3}{|c|}{ Ear position (\%) } & \multicolumn{3}{|c|}{$\begin{array}{l}\text { Resistance to downy } \\
\text { mildew disease }(\%)\end{array}$} & \multicolumn{3}{|c|}{$\begin{array}{c}\text { Resistance to late wilt } \\
\text { disease }(\%)\end{array}$} \\
\hline & & $Y_{1}$ & $\mathbf{Y}_{2}$ & Comb. & $Y_{1}$ & $\mathbf{Y}_{2}$ & Comb. & $Y_{1}$ & $\mathbf{Y}_{2}$ & Comb. \\
\hline $\mathrm{P}$ & & 0.505 & 0.683 & 0.594 & -2.531 & -1.375 & -1.953 & 0.646 & 0.449 & 0.548 \\
\hline $\mathrm{P}$ & & $-4.152 * *$ & $-2.596 * *$ & $-3.374 * *$ & $-8.009 * *$ & $-3.075^{*}$ & $-5.542 * *$ & -1.451 & 0.690 & -0.380 \\
\hline $\mathrm{P}$ & & $7.243 * *$ & $5.451 * *$ & $6.347 * *$ & -0.069 & $4.844 * *$ & 2.388 & $11.393 * *$ & 0.596 & $5.995 * *$ \\
\hline $\mathrm{P}$ & & 0.492 & $0.910 *$ & $0.701 *$ & 2.175 & -1.459 & 0.358 & $6.887^{* *}$ & $3.221 *$ & $5.054^{* *}$ \\
\hline $\mathrm{P}$ & & $0.841 * *$ & 0.364 & 0.603 & -0.481 & -1.525 & -1.003 & $-7.626^{* *}$ & $3.740 *$ & -1.943 \\
\hline $\mathrm{P}$ & & $1.074 * *$ & 0.669 & $0.872 *$ & $4.916^{* *}$ & 0.866 & $2.891 *$ & $-6.310 * *$ & 1.746 & -2.282 \\
\hline $\mathrm{P}$ & & $-4.771 * *$ & $-3.633 * *$ & $-4.202 * *$ & $3.828^{* *}$ & $3.238 *$ & $3.533 *$ & $-5.610 * *$ & $-3.126^{*}$ & $-4.368^{* *}$ \\
\hline $\mathrm{P}$ & & $-4.637 * *$ & $-3.493 * *$ & $-4.065^{* *}$ & 1.447 & -0.609 & 0.419 & $-8.079 * *$ & $-8.860 * *$ & $-8.469 * *$ \\
\hline $\mathrm{P}$ & & 0.108 & $-1.323 * *$ & -0.607 & -1.850 & $-3.591 *$ & -2.720 & 0.896 & $-3.669 *$ & -1.387 \\
\hline$P_{1}$ & & $3.297 * *$ & $2.967 * *$ & $3.132 * *$ & 0.575 & 2.688 & 1.631 & $9.253 * *$ & $5.212^{* *}$ & $7.232 * *$ \\
\hline \multirow[t]{2}{*}{ L.S.D gi } & 0.05 & 0.633 & 0.732 & 0.683 & 2.847 & 2.817 & 2.832 & 2.607 & 3.041 & 2.824 \\
\hline & 0.01 & 0.834 & 0.963 & 0.899 & 3.747 & 3.709 & 3.728 & 3.432 & 4.003 & 3.718 \\
\hline L.S.D & 0.05 & 0.944 & 1.091 & 1.018 & 4.244 & 4.2 & 4.222 & 3.886 & 4.533 & 4.210 \\
\hline gi-gj & 0.01 & 1.243 & 1.436 & 1.340 & 5.586 & 5.528 & 5.557 & 5.116 & 5.967 & 5.542 \\
\hline \multicolumn{11}{|c|}{ 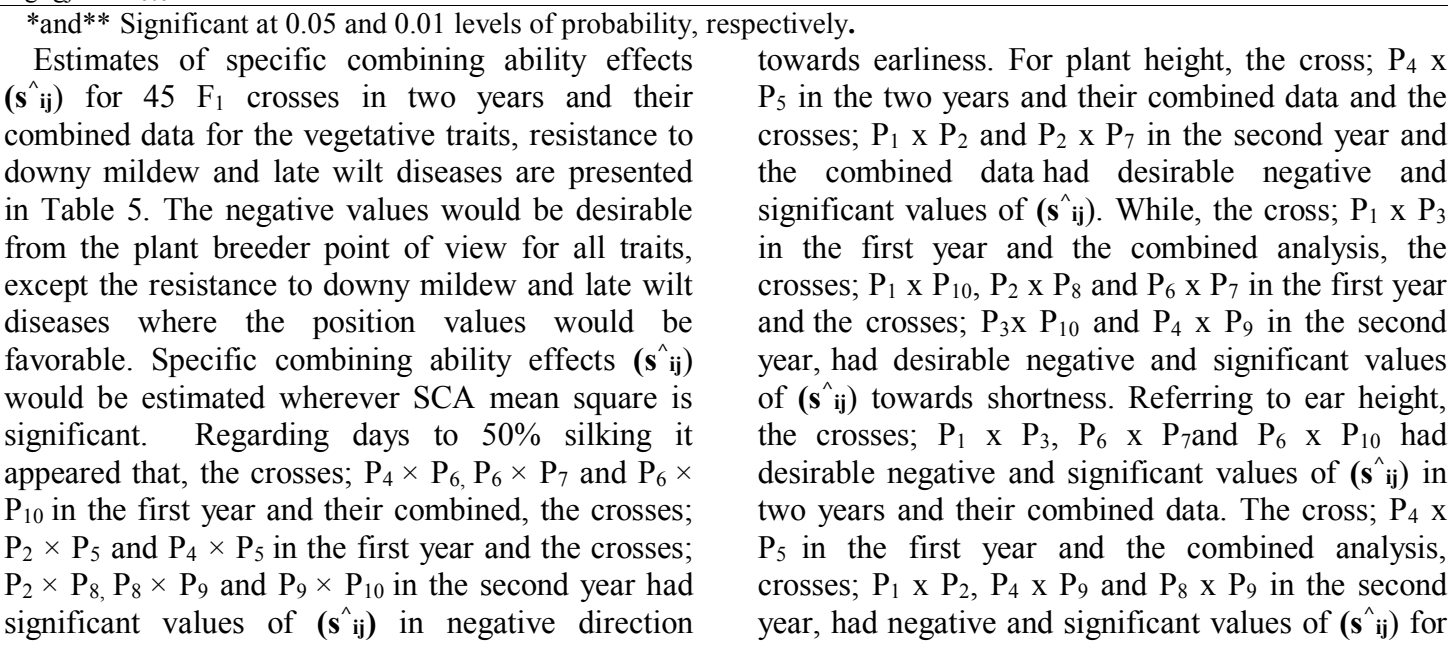 } \\
\hline
\end{tabular}


low ear height. For ear position, the crosses; $\mathrm{P}_{1} \times \mathrm{P}_{3}$ and $\mathrm{P}_{6} \times \mathrm{P}_{7}$ had desirable negative and significant values of $\left(\mathbf{s}_{\mathbf{i j}}\right)$ in the two years and their combined. While, the crosses; $\mathrm{P}_{2} \times \mathrm{P}_{9}$ and $\mathrm{P}_{4} \times \mathrm{P}_{6}$ in the first year and the combined and the crosses; $\mathrm{P}_{5} \times \mathrm{P}_{8}, \mathrm{P}_{6} \mathrm{x}$ $\mathrm{P}_{10}$ and $\mathrm{P}_{9} \mathrm{X} \mathrm{P}_{10}$ in the first year, had negative and significant values of $\left(\mathbf{s}^{\wedge} \mathbf{i j}\right)$ for low ear position. Concerning resistance to downy mildew disease, the cross; $\mathrm{P}_{2} \mathrm{X} \mathrm{P}_{4}$ in the two years and their combined analysis and the cross; $\mathrm{P}_{2} \times \mathrm{P}_{3}$ in the first year and the combined data had positive and significant values of $\left(\mathbf{s}_{\mathbf{i j}}\right)$. While, the cross; $\mathrm{P}_{1} \times \mathrm{P}_{5}$ in the first year and crosses; $\mathrm{P}_{5} \times \mathrm{P}_{9}, \mathrm{P}_{8} \times \mathrm{P}_{9}$ in the second year had desirable positive and significant values of $\left(\mathbf{s}^{\wedge} \mathbf{i j}\right)$ for resistance to downy mildew disease. For resistance to late wilt disease, the cross; $\mathrm{P}_{8} \times \mathrm{P}_{10}$ in the two years and their combined analysis and the crosses; $\mathrm{P}_{1} \times \mathrm{P}_{7}, \mathrm{P}_{1} \times \mathrm{P}_{9}$ and $\mathrm{P}_{6} \times \mathrm{P}_{8}$ in the first year and the combined data had positive and significant values of ( $\left.\mathbf{s}_{\mathbf{i j}}\right)$. The crosses; $\mathrm{P}_{1} \times \mathrm{P}_{2}, \mathrm{P}_{2} \times \mathrm{P}_{5}, \mathrm{P}_{2} \times \mathrm{P}_{7}$, $\mathrm{P}_{3} \times \mathrm{P}_{8}, \mathrm{P}_{4} \times \mathrm{P}_{5}$ and $\mathrm{P}_{4} \times \mathrm{P}_{6}$ in the first year and the cross; $\mathrm{P}_{6} \times \mathrm{P}_{7}$ in the second year, had desirable and significant values of $\left(\mathbf{s}^{\wedge} \mathbf{i j}\right)$ for resistance to late wilt disease. From the obtained results, it might be inferred that, the single crosses; $\mathrm{P}_{1} \times \mathrm{P}_{3}, \mathrm{P}_{6} \times \mathrm{P}_{7}$ and $\mathrm{P}_{4} \times \mathrm{P}_{5}$ had favorable allels for good plant type with earliness, indicating that these new hybrids might be promising in future maize breeding program.

Superiority percentages of the $45 \mathrm{~F}_{1}$ crosses relative to two checks SC 162 and SC 168 for the vegetative traits, resistance to downy mildew and late wilt diseases as an average of two years were presented in Table 6. It might be noted that, negative values would be desired for days to $50 \%$ silking, plant height, ear height and ear position while the positive values would be favored for the other studied traits, i.e., resistance to downy mildew and late wilt diseases.

plant height, ear height and ear position, except for plant height with regard to the check cultivar SC168, where, the values in this concern did not reach the level of significant. However, the first group of crosses could be used as new hybrid.
It was noted that the crosses; $\mathrm{P}_{1} \times \mathrm{P}_{2}, \mathrm{P}_{1} \times \mathrm{P}_{8}, \mathrm{P}_{2} \mathrm{x}$ $\mathrm{P}_{4}, \mathrm{P}_{2} \times \mathrm{P}_{8}, \mathrm{P}_{3} \times \mathrm{P}_{8}, \mathrm{P}_{4} \times \mathrm{P}_{8}, \mathrm{P}_{5} \times \mathrm{P}_{8}, \mathrm{P}_{6} \times \mathrm{P}_{8}, \mathrm{P}_{7} \times \mathrm{P}_{8}$ $\mathrm{P}_{8} \times \mathrm{P}_{9}$ and $\mathrm{P}_{8} \times \mathrm{P}_{10}$ had significant superiority percentage in negative direction relative the two checks i.e.; SC 162 and SC168 for days to $50 \%$ silking, plant height, ear height and ear position. While, the crosses; $\mathrm{P}_{1} \times \mathrm{P}_{4}, \mathrm{P}_{1} \times \mathrm{P}_{5}, \mathrm{P}_{1} \times \mathrm{P}_{7}, \mathrm{P}_{2} \times \mathrm{P}_{5}$, $\mathrm{P}_{2} \times \mathrm{P}_{7}, \mathrm{P}_{1} \times \mathrm{P}_{9}, \mathrm{P}_{2} \times \mathrm{P}_{10}, \mathrm{P}_{4} \times \mathrm{P}_{6}, \mathrm{P}_{4} \times \mathrm{P}_{7}, \mathrm{P}_{4} \times \mathrm{P}_{9}, \mathrm{P}_{5}$ $\times \mathrm{P}_{7}, \mathrm{P}_{6} \times \mathrm{P}_{7}, \mathrm{P}_{7} \times \mathrm{P}_{9}$ and $\mathrm{P}_{7} \times \mathrm{P}_{10}$ had superiority percentages in negative direction relative the two check cultivars for days to $50 \%$ silking, cultivars characterized by earliness and short stem after more evaluation in different location and years. While, the second group of crosses could be used as new hybrid cultivars characterized by earliness, where its results referred to its superiority with regard to the two cultivar checks in this respect. Concerning to the resistance to downy mildew and late wilt diseases, the results listed in Table 6 revealed that there is no significant value in positive direction in all crosses over either the check cultivar SC162 or the check cultivar SC168 for traits, which indicated the present genetic material failed to regenerate a new genotype more resistant than the two check cultivars used in the study. 
Table 5: Estimates of specific combining ability effects $\left(\hat{s}_{\mathrm{ij}}\right)$ for $45 \mathrm{~F}_{1}$ crosses in two years and their combined data for the vegetative traits, resistance to downy mildew and late wilt diseases.

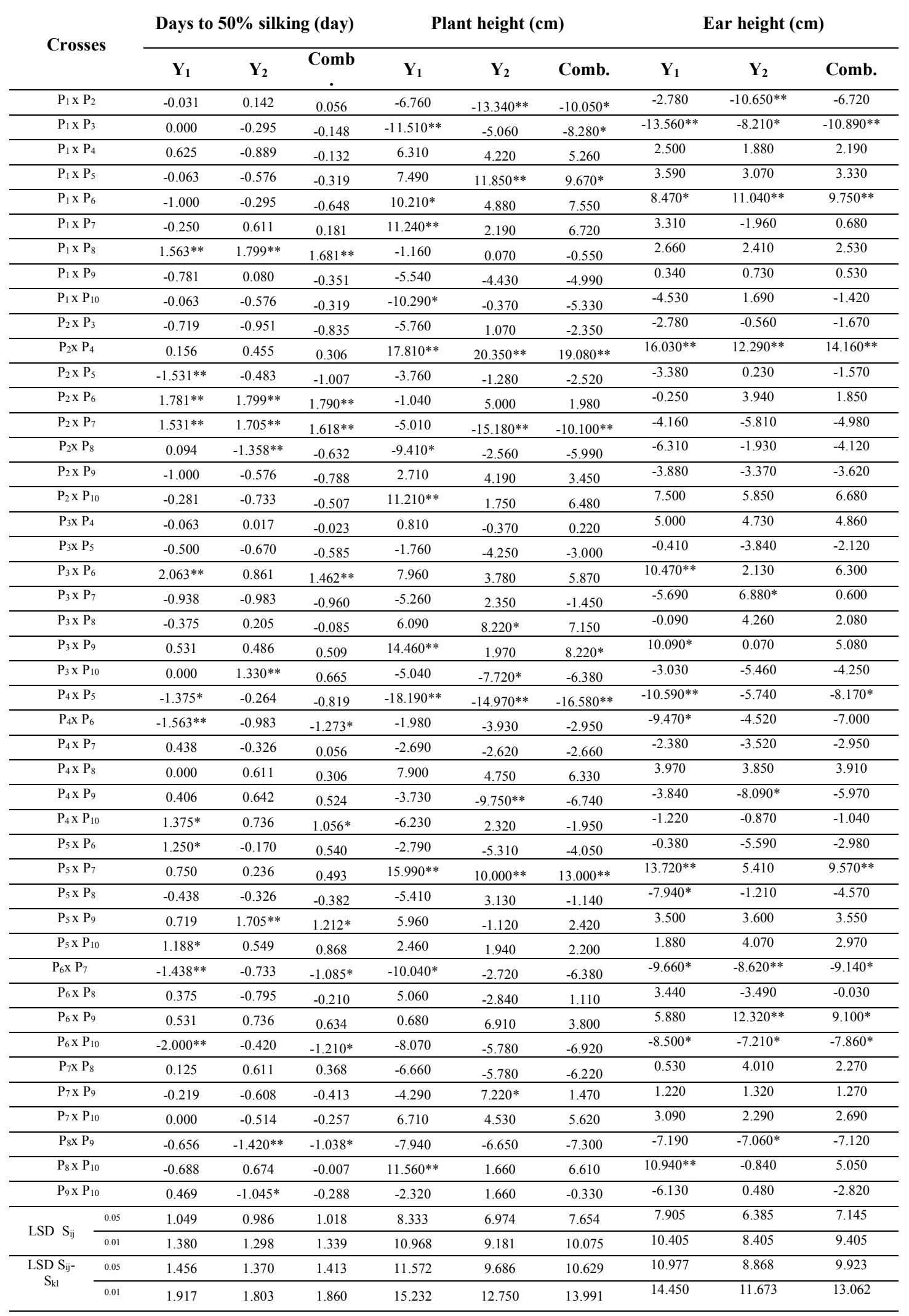


*and** Significant at 0.05 and 0.01 levels of probability, respectively.

Table 5:Cont.

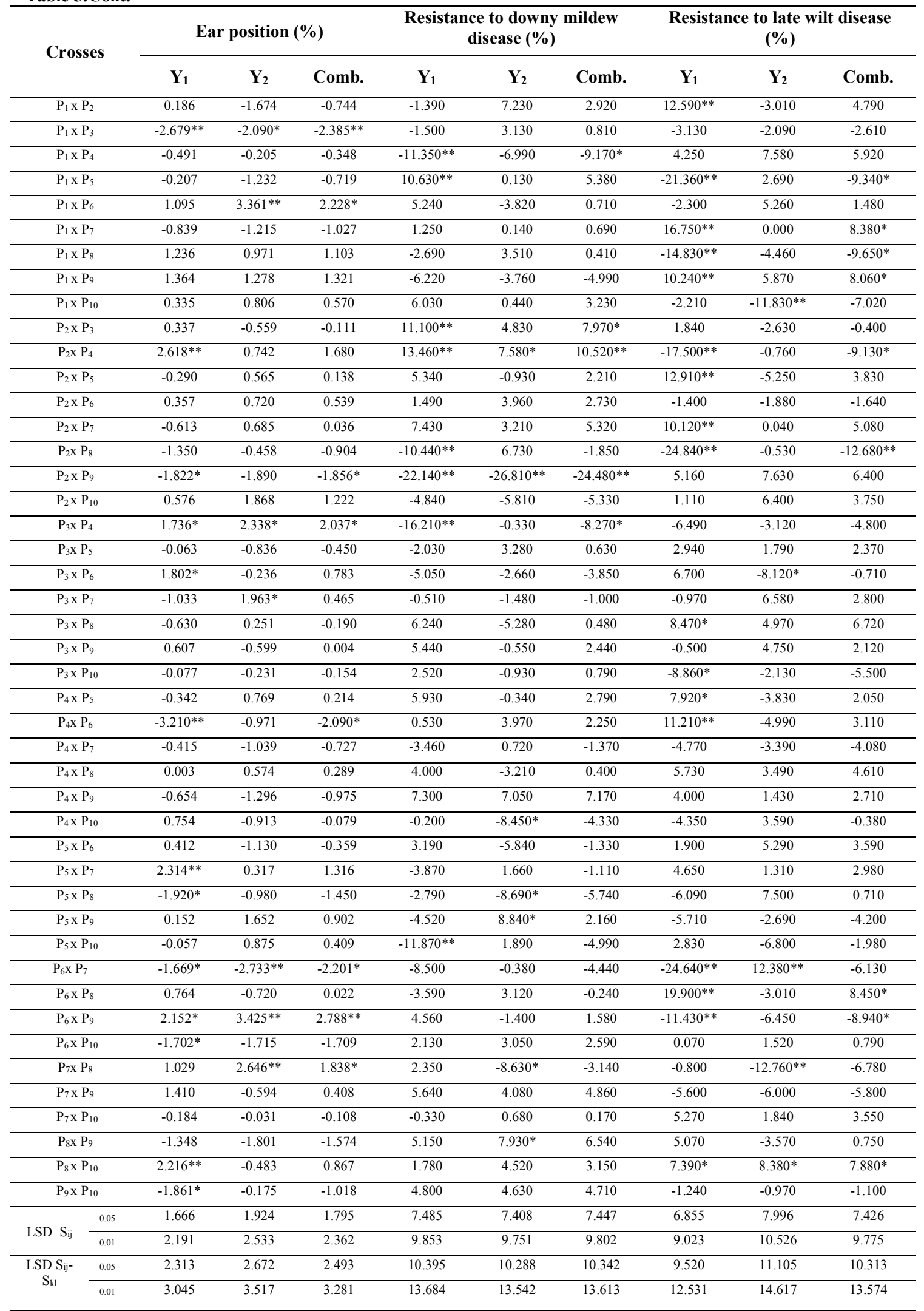


*and** Significant at 0.05 and 0.01 levels of probability, respectively.

Table 6:Superiority percentages of the $45 \mathrm{~F}_{1}$ crosses relative to two checks SC 162 and SC 168 for the vegetative traits, resistance to downy mildew and late wilt diseases as an average of two years.

\begin{tabular}{|c|c|c|c|c|c|c|c|}
\hline \multirow{2}{*}{\multicolumn{2}{|c|}{ Crosses }} & \multicolumn{2}{|c|}{$\begin{array}{c}\text { Days to } 50 \% \text { silking } \\
\text { (day) }\end{array}$} & \multicolumn{2}{|c|}{ Plant height (cm) } & \multicolumn{2}{|c|}{ Ear height (cm) } \\
\hline & & SC 162 & SC 168 & SC 162 & SC 162 & SC 168 & SC 162 \\
\hline & $\mathrm{P}_{1} \times \mathrm{P}_{2}$ & $-5.93 * *$ & $-4.29 * *$ & $-23.23 * *$ & $-4.57 *$ & $-32.47 * *$ & $-24.60 * *$ \\
\hline & $\mathrm{P}_{1} \times \mathrm{P}_{3}$ & $-2.30 *$ & -0.59 & $-24.75 * *$ & $-6.45 * *$ & $-22.68 * *$ & $-13.67 * *$ \\
\hline & $\mathrm{P}_{1} \times \mathrm{P}_{4}$ & $-5.16 * *$ & $-3.51 * *$ & $-20.28 * *$ & -0.89 & $-23.33 * *$ & $-14.39 * *$ \\
\hline & $\mathrm{P}_{1} \times \mathrm{P}_{5}$ & $-4.40 * *$ & $-2.73 * *$ & $-13.49 * *$ & $7.54 * *$ & $-17.64 * *$ & $-8.04 * *$ \\
\hline & $\mathrm{P}_{1} \times \mathrm{P}_{6}$ & $-4.21 * *$ & $-2.54 * *$ & $-15.13 * *$ & $5.51 * *$ & $-14.33 * *$ & -4.34 \\
\hline & $\mathrm{P}_{1} \times \mathrm{P}_{7}$ & $-3.83 * *$ & $-2.15^{*}$ & $-16.93 * *$ & 3.28 & $-28.66^{* *}$ & $-20.34 * *$ \\
\hline & $\mathrm{P}_{1} \times \mathrm{P}_{8}$ & $-6.31 * *$ & $-4.68 * *$ & $-28.18 * *$ & $-10.72 * *$ & $-35.28 * *$ & $-27.73 * *$ \\
\hline & $\mathrm{P}_{1} \times \mathrm{P}_{9}$ & $-4.40 * *$ & $-2.73 * *$ & $-21.72 * *$ & -2.68 & $-24.26 * *$ & $-15.43 * *$ \\
\hline & $\mathrm{P}_{1} \times \mathrm{P}_{10}$ & $-4.78 * *$ & $-3.12 * *$ & $-22.08 * *$ & -3.13 & $-20.38 * *$ & $-11.09 * *$ \\
\hline & $\mathrm{P}_{2} \times \mathrm{P}_{3}$ & $-6.31 * *$ & $-4.68 * *$ & $-20.48 * *$ & -1.14 & $-20.81 * *$ & $-11.58 * *$ \\
\hline & $\mathrm{P}_{2} \times \mathrm{P}_{4}$ & $-7.46 * *$ & $-5.85 * *$ & $-13.49 * *$ & $7.54 * *$ & $-19.87 * *$ & $-10.53 * *$ \\
\hline & $\mathrm{P}_{2} \times \mathrm{P}_{5}$ & $-8.43 * *$ & $-6.83 * *$ & $-15.01 * *$ & $5.66 * *$ & $-23.90 * *$ & $-15.03 * *$ \\
\hline & $\mathrm{P}_{2} \times \mathrm{P}_{6}$ & $-3.45 * *$ & -1.76 & $-14.53 * *$ & $6.25 * *$ & $-22.32 * *$ & $-13.26 * *$ \\
\hline & $\mathrm{P}_{2} \times \mathrm{P}_{7}$ & $-4.60 * *$ & $-2.93 * *$ & $-19.92 * *$ & -0.45 & $-35.35 * *$ & $-27.81 * *$ \\
\hline & $\mathrm{P}_{2} \mathrm{X} \mathrm{P}_{8}$ & $-12.83 * *$ & $-11.31 * *$ & $-27.54 * *$ & $-9.93 * *$ & $-42.55 * *$ & $-35.85 * *$ \\
\hline & $\mathrm{P}_{2} \times \mathrm{P}_{9}$ & $-8.05 * *$ & $-6.44 * *$ & $-16.65 * *$ & 3.62 & $-30.10 * *$ & $-21.95 * *$ \\
\hline & $\mathrm{P}_{2} \times \mathrm{P}_{10}$ & $-8.05 * *$ & $-6.44 * *$ & $-15.93 * *$ & $4.51 *$ & $-19.15 * *$ & $-9.73 * *$ \\
\hline & $\mathrm{P}_{3} \times \mathrm{P}_{4}$ & $-4.02 * *$ & $-2.34 *$ & $-21.60 * *$ & -2.53 & $-13.03 * *$ & -2.89 \\
\hline & $\mathrm{P}_{3} \times \mathrm{P}_{5}$ & $-3.83 * *$ & $-2.15^{*}$ & $-17.25 * *$ & 2.88 & $-12.03 * *$ & -1.77 \\
\hline & $\mathrm{P}_{3} \times \mathrm{P}_{6}$ & 0.00 & 1.75 & $-15.37 * *$ & $5.21 * *$ & $-7.56 * *$ & 3.22 \\
\hline & $\mathrm{P}_{3} \times \mathrm{P}_{7}$ & $-4.60 * *$ & $-2.93 * *$ & $-19.24 * *$ & 0.40 & $-19.94 * *$ & $-10.61 * *$ \\
\hline & $\mathrm{P}_{3} \times \mathrm{P}_{8}$ & $-8.05 * *$ & $-6.44 * *$ & $-25.43 * *$ & $-7.30 * *$ & $-26.78 * *$ & $-18.25^{* *}$ \\
\hline & $\mathrm{P}_{3} \times \mathrm{P}_{9}$ & $-2.10 *$ & -0.39 & $-17.21 * *$ & 2.93 & $-12.89 * *$ & -2.73 \\
\hline & $\mathrm{P}_{3} \times \mathrm{P}_{10}$ & $-2.30 *$ & -0.59 & $-22.12 * *$ & -3.18 & $-13.25 * *$ & -3.14 \\
\hline & $\mathrm{P}_{4} \times \mathrm{P}_{5}$ & $-7.08 * *$ & $-5.46 * *$ & $-21.44 * *$ & -2.33 & $-23.69 * *$ & $-14.79 * *$ \\
\hline & $\mathrm{P}_{4} \times \mathrm{P}_{6}$ & $-7.08 * *$ & $-5.46^{* *}$ & $-18.04 * *$ & 1.89 & $-23.40 * *$ & $-14.47 * *$ \\
\hline & $\mathrm{P}_{4} \times \mathrm{P}_{7}$ & $-5.93 * *$ & $-4.29 * *$ & $-19.48 * *$ & 0.10 & $-30.17 * *$ & $-22.03 * *$ \\
\hline & $\mathrm{P}_{4} \times \mathrm{P}_{8}$ & $-10.34 * *$ & $-8.78 * *$ & $-25.55 * *$ & $-7.44 * *$ & $-33.91 * *$ & $-26.21 * *$ \\
\hline & $\mathrm{P}_{4} \times \mathrm{P}_{9}$ & $-4.98 * *$ & $-3.32 * *$ & $-21.84 * *$ & -2.83 & $-27.43 * *$ & $-18.97 * *$ \\
\hline & $\mathrm{P}_{4} \times \mathrm{P}_{10}$ & $-4.60 * *$ & $-2.93 * *$ & $-20.56 * *$ & -1.24 & $-19.58 * *$ & $-10.21 * *$ \\
\hline & $\mathrm{P}_{5} \times \mathrm{P}_{6}$ & $-3.25 * *$ & -1.56 & $-13.01 * *$ & $8.14 * *$ & $-16.06^{* *}$ & $-6.27 *$ \\
\hline & $\mathrm{P}_{5} \times \mathrm{P}_{7}$ & $-4.21 * *$ & $-2.54 * *$ & $-9.10 * *$ & $13.00 * *$ & $-17.93 * *$ & $-8.36 * *$ \\
\hline & $\mathrm{P}_{5} \times \mathrm{P}_{8}$ & $-10.34 * *$ & $-8.78 * *$ & $-22.56 * *$ & $-3.72 *$ & $-33.77 * *$ & $-26.05 * *$ \\
\hline & $\mathrm{P}_{5} \times \mathrm{P}_{9}$ & $-2.87 * *$ & -1.17 & $-13.53 * *$ & $7.49 * *$ & $-16.92 * *$ & $-7.23 * *$ \\
\hline & $\mathrm{P}_{5} \times \mathrm{P}_{10}$ & $-3.83 * *$ & $-2.15^{*}$ & $-13.85 * *$ & $7.09 * *$ & $-12.24 * *$ & -2.01 \\
\hline & $\mathrm{P}_{6} \times \mathrm{P}_{7}$ & $-5.93 * *$ & $-4.29 * *$ & $-16.25 * *$ & $4.12 *$ & $-29.09 * *$ & $-20.82 * *$ \\
\hline & $\mathrm{P}_{6} \times \mathrm{P}_{8}$ & $-9.38 * *$ & $-7.80 * *$ & $-22.80 * *$ & $-4.02 *$ & $-31.54 * *$ & $-23.55 * *$ \\
\hline & $\mathrm{P}_{6} \times \mathrm{P}_{9}$ & $-3.07 * *$ & -1.37 & $-14.05 * *$ & $6.85 * *$ & $-14.11 * *$ & -4.10 \\
\hline & $\mathrm{P}_{6} \times \mathrm{P}_{10}$ & $-6.31 * *$ & $-4.68 * *$ & $-17.72 * *$ & 2.28 & $-18.86^{* *}$ & $-9.41 * *$ \\
\hline & $\mathrm{P}_{7} \times \mathrm{P}_{8}$ & $-9.38 * *$ & $-7.80 * *$ & $-26.67 * *$ & $-8.83 * *$ & $-39.31 * *$ & $-32.23 * *$ \\
\hline & $\mathrm{P}_{7} \times \mathrm{P}_{9}$ & $-5.55 * *$ & $-3.90 * *$ & $-16.33 * *$ & $4.02 *$ & $-27.72 * *$ & $-19.29 * *$ \\
\hline & $\mathrm{P}_{7} \times \mathrm{P}_{10}$ & $-5.75 * *$ & $-4.10 * *$ & $-15.25 * *$ & $5.36 * *$ & $-21.89 * *$ & $-12.78 * *$ \\
\hline & $\mathrm{P}_{8} \times \mathrm{P}_{9}$ & $-11.30 * *$ & $-9.75 * *$ & $-28.07 * *$ & $-10.57 * *$ & $-40.25 * *$ & $-33.28 * *$ \\
\hline & $\mathrm{P}_{8} \times \mathrm{P}_{10}$ & $-10.15^{* *}$ & $-8.58 * *$ & $-23.87 * *$ & $-5.36 * *$ & $-28.22 * *$ & $-19.86 * *$ \\
\hline & $\mathrm{P}_{9} \times \mathrm{P}_{10}$ & $-5.55 * *$ & $-3.90 * *$ & $-18.20 * *$ & 1.69 & $-20.59 * *$ & $-11.33 * *$ \\
\hline \multirow{2}{*}{ LSD } & 0.05 & \multicolumn{2}{|c|}{1.148} & \multicolumn{2}{|c|}{8.747} & \multicolumn{2}{|c|}{8.240} \\
\hline & 0.01 & \multicolumn{2}{|c|}{1.512} & \multicolumn{2}{|c|}{11.514} & \multicolumn{2}{|c|}{10.847} \\
\hline
\end{tabular}


*and** Significant at 0.05 and 0.01 levels of probability, respectively.

Table 6:Cont.

\begin{tabular}{|c|c|c|c|c|c|c|c|}
\hline \multirow{2}{*}{\multicolumn{2}{|c|}{ Crosses }} & \multicolumn{2}{|c|}{ Ear position (\%) } & \multicolumn{2}{|c|}{$\begin{array}{l}\text { Resistance to downy } \\
\text { mildew disease }(\%)\end{array}$} & \multicolumn{2}{|c|}{$\begin{array}{c}\text { Resistance to late wilt } \\
\text { disease }(\%)\end{array}$} \\
\hline & & SC 162 & SC 168 & SC 162 & & SC 162 & SC 168 \\
\hline & $\mathrm{P}_{1} \times \mathrm{P}_{2}$ & $-11.93 * *$ & $-20.80 * *$ & -1.19 & -3.11 & -1.94 & 4.38 \\
\hline & $\mathrm{P}_{1} \times \mathrm{P}_{3}$ & 2.65 & $-7.69 * *$ & 1.45 & -0.52 & -1.94 & 4.38 \\
\hline & $\mathrm{P}_{1} \times \mathrm{P}_{4}$ & $-3.84 *$ & $-13.53 * *$ & -4.49 & -6.34 & 1.67 & 8.22 \\
\hline & $\mathrm{P}_{1} \times \mathrm{P}_{5}$ & $-4.69^{*}$ & $-14.30 * *$ & 2.91 & 0.91 & $-21.11 * *$ & $-16.02 * *$ \\
\hline & $\mathrm{P}_{1} \times \mathrm{P}_{6}$ & 1.10 & $-9.09 * *$ & 3.17 & 1.17 & -5.92 & 0.14 \\
\hline & $\mathrm{P}_{1} \times \mathrm{P}_{7}$ & $-13.93 * *$ & $-22.61 * *$ & 2.77 & 0.78 & -3.99 & 2.19 \\
\hline & $\mathrm{P}_{1} \times \mathrm{P}_{8}$ & $-9.84 * *$ & $-18.92 * *$ & 1.18 & -0.78 & $-25.62 * *$ & $-20.82 * *$ \\
\hline & $\mathrm{P}_{1} \times \mathrm{P}_{9}$ & -3.19 & $-12.95 * *$ & -8.86 & $-10.62 *$ & -0.65 & 5.75 \\
\hline & $\mathrm{P}_{1} \times \mathrm{P}_{10}$ & 2.18 & $-8.11 * *$ & 3.43 & 1.43 & -5.54 & 0.55 \\
\hline & $\mathrm{P}_{2} \times \mathrm{P}_{3}$ & -0.40 & $-10.43 * *$ & 4.35 & 2.33 & -2.97 & 3.29 \\
\hline & $\mathrm{P}_{2} \times \mathrm{P}_{4}$ & $-7.35 * *$ & $-16.68 * *$ & 4.88 & 2.85 & $-10.30 *$ & -4.52 \\
\hline & $\mathrm{P}_{2} \times \mathrm{P}_{5}$ & $-10.32 * *$ & $-19.36 * *$ & -1.06 & -2.97 & -4.63 & 1.51 \\
\hline & $\mathrm{P}_{2} \times \mathrm{P}_{6}$ & $-9.11 * *$ & $-18.27 * *$ & 1.59 & -0.38 & -7.34 & -1.37 \\
\hline & $\mathrm{P}_{2} \times \mathrm{P}_{7}$ & $-19.17 * *$ & $-27.31 * *$ & 3.83 & 1.81 & -6.05 & 0.00 \\
\hline & $\mathrm{P}_{2} \times \mathrm{P}_{8}$ & $-20.61 * *$ & $-28.61 * *$ & -4.76 & -6.60 & $-32.69 * *$ & $-28.35 * *$ \\
\hline & $\mathrm{P}_{2} \times \mathrm{P}_{9}$ & $-16.10 * *$ & $-24.55 * *$ & $-37.78 * *$ & $-38.98 * *$ & -2.57 & 3.70 \\
\hline & $\mathrm{P}_{2} \times \mathrm{P}_{10}$ & $-3.79 *$ & $-13.49 * *$ & -4.10 & -5.96 & 1.67 & 8.22 \\
\hline & $\mathrm{P}_{3} \times \mathrm{P}_{4}$ & $10.83 * *$ & -0.34 & -3.70 & -5.56 & -2.19 & 4.11 \\
\hline & $\mathrm{P}_{3} \times \mathrm{P}_{5}$ & $6.17 * *$ & $-4.53 * *$ & 2.38 & 0.39 & -1.54 & 4.80 \\
\hline & $\mathrm{P}_{3} \times \mathrm{P}_{6}$ & $8.88 * *$ & -2.09 & 1.32 & -0.64 & -4.25 & 1.92 \\
\hline & $\mathrm{P}_{3} \times \mathrm{P}_{7}$ & -0.85 & $-10.84 * *$ & 4.49 & 2.47 & -2.71 & 3.56 \\
\hline & $\mathrm{P}_{3} \times \mathrm{P}_{8}$ & -1.79 & $-11.68 * *$ & 3.83 & 1.81 & -2.71 & 3.56 \\
\hline & $\mathrm{P}_{3} \times \mathrm{P}_{9}$ & $4.80 *$ & $-5.76 * *$ & 2.50 & 0.52 & -1.16 & 5.21 \\
\hline & $\mathrm{P}_{3} \times \mathrm{P}_{10}$ & $11.26^{* *}$ & 0.05 & 4.35 & 2.33 & -0.13 & 6.30 \\
\hline & $\mathrm{P}_{4} \times \mathrm{P}_{5}$ & -2.82 & $-12.61 * *$ & 2.50 & 0.52 & -1.68 & 4.66 \\
\hline & $\mathrm{P}_{4} \times \mathrm{P}_{6}$ & $-6.50 * *$ & $-15.92 * *$ & 5.02 & 2.98 & -2.19 & 4.11 \\
\hline & $\mathrm{P}_{4} \times \mathrm{P}_{7}$ & $-13.19 * *$ & $-21.94 * *$ & 3.03 & 1.04 & -7.60 & -1.64 \\
\hline & $\mathrm{P}_{4} \times \mathrm{P}_{8}$ & $-11.12^{* *}$ & $-20.07 * *$ & 1.18 & -0.78 & -3.74 & 2.47 \\
\hline & $\mathrm{P}_{4} \times \mathrm{P}_{9}$ & $-7.15 * *$ & $-16.50 * *$ & 4.35 & 2.33 & -1.54 & 4.80 \\
\hline & $\mathrm{P}_{4} \times \mathrm{P}_{10}$ & 1.21 & $-8.99 * *$ & 0.13 & -1.81 & 2.06 & 8.64 \\
\hline & $\mathrm{P}_{5} \times \mathrm{P}_{6}$ & -3.56 & $-13.27 * *$ & 0.53 & -1.42 & -7.86 & -1.92 \\
\hline & $\mathrm{P}_{5} \times \mathrm{P}_{7}$ & $-9.69 * *$ & $-18.79 * *$ & 2.91 & 0.91 & -8.49 & -2.60 \\
\hline & $\mathrm{P}_{5} \times \mathrm{P}_{8}$ & $-14.42 * *$ & $-23.04 * *$ & -1.99 & -3.89 & $-18.92 * *$ & $-13.70 * *$ \\
\hline & $\mathrm{P}_{5} \times \mathrm{P}_{9}$ & $-3.93 *$ & $-13.62 * *$ & -1.06 & -2.97 & $-11.58 * *$ & -5.88 \\
\hline & $\mathrm{P}_{5} \times \mathrm{P}_{10}$ & 1.91 & $-8.36 * *$ & -3.31 & -5.18 & -2.57 & 3.70 \\
\hline & $\mathrm{P}_{6} \mathrm{x} \mathrm{P}_{7}$ & $-15.54 * *$ & $-24.05 * *$ & 3.43 & 1.43 & $-27.16^{* *}$ & $-22.47 * *$ \\
\hline & $\mathrm{P}_{6} \times \mathrm{P}_{8}$ & $-11.28 * *$ & $-20.22 * *$ & 3.56 & 1.55 & $-9.27 *$ & -3.42 \\
\hline & $\mathrm{P}_{6} \times \mathrm{P}_{9}$ & -0.05 & $-10.13 * *$ & 1.71 & -0.26 & $-18.41 * *$ & $-13.15 * *$ \\
\hline & $\mathrm{P}_{6} \times \mathrm{P}_{10}$ & -1.43 & $-11.36^{* *}$ & 5.67 & 3.63 & -2.97 & 3.29 \\
\hline & $\mathrm{P}_{7} \mathrm{x} \mathrm{P}_{8}$ & $-17.16^{* *}$ & $-25.51 * *$ & 0.39 & -1.55 & $-29.86 * *$ & $-25.34 * *$ \\
\hline & $\mathrm{P}_{7} \times \mathrm{P}_{9}$ & $-13.50 * *$ & $-22.22 * *$ & 5.15 & 3.11 & $-16.35 * *$ & $-10.96 *$ \\
\hline & $\mathrm{P}_{7} \times \mathrm{P}_{10}$ & $-7.69 * *$ & $-16.99 * *$ & 4.88 & 2.85 & -0.51 & 5.90 \\
\hline & $\mathrm{P}_{8} \mathrm{x} \mathrm{P}_{9}$ & $-16.84 * *$ & $-25.22 * *$ & 4.62 & 2.59 & $-15.83 * *$ & $-10.41^{*}$ \\
\hline & $\mathrm{P}_{8} \times \mathrm{P}_{10}$ & $-5.68 * *$ & $-15.19 * *$ & 4.76 & 2.73 & -1.42 & 4.93 \\
\hline & $\mathrm{P}_{9} \times \mathrm{P}_{10}$ & -2.85 & $-12.64 * *$ & 4.09 & 2.07 & -2.45 & 3.84 \\
\hline \multirow{2}{*}{ LSD } & 0.05 & \multicolumn{2}{|c|}{2.043} & \multicolumn{2}{|c|}{8.495} & \multicolumn{2}{|c|}{8.368} \\
\hline & 0.01 & \multicolumn{2}{|c|}{2.689} & \multicolumn{2}{|c|}{11.182} & \multicolumn{2}{|c|}{11.015} \\
\hline
\end{tabular}

*and** Significant at 0.05 and 0.01 levels of probability, respectively. 


\section{REFERENCES}

Abd El-Hadi, A.H.; Kawther S. Kash; A.A. ElShenawy and I.A. El-Gazzar (2005). Combining ability and heterosis in maize (Zea mays L.). Egypt. J. Genet. Cytol., 34: 123-134.

Akanda, M.A.L.; M.F. Alam and M.M. Hossain (2007). Combining ability for grain yield and its components of subtropical maize (Zea mays L.) inbred lines. Annals of Bangladesh Agriculture, 11(1): 77-85.

Amer, E. A.; A. A. El-Shenawy and H. E. Mosa (2002). Evaluation of some new inbred lines of maize for combining ability. Annals of Agricultural Science, Moshtohor, 40(2): 791802.

Amer, E.A.; H.E. Mosa and A.A. Motawei (2002). Genetic analysis for grain yield, downy mildew, late wilt and kernel rot disease on maize. J. Agric. Sci. Mansoura Univ. 27: 1965- 1974.

Bartlett, M.S. (1937). Properties of sufficiency and statistical test. Proc. Roy. Soc. Lond., 160A, 268-282.

El-Gazzar, I.A.I (2015). Combining ability for growth, yield and its attributes and resistance to downy mildew disease in maize. J. Agric. Res. Kafr El-Sheikh Univ., 41(2) 2015.

El-Itriby, H.A.; M.N. Khamis; R.M. El-Demerdash and H.A. El-Shafey (1984). Inheritance of resistance to late wilt (Cephalosporium maydis) in maize. Proc. $2^{\text {nd }}$ Mediterranean Conf. Genet., Cairo, March: 29-44.

El-Shamarka, Sh.A.; M. Abdel-Sattar and M.ElNahas (2015). Heterosis and combining ability for yield and its components through diallel cross analysis in maize (Zea mays L.). Alex. J. Agric.Res.60(2):87-94

El-Shenawy, A.A. (1995). Breeding for disease resistance in maize. Ph.D. Thesis, Fac. Agric. Minufiya Univ., Egypt.

Galal, A.A.; M.M. EL-Rouby and A.M. Gad (1979). Genetic analysis of resistance to late wilt (Cephalosporium maydis) in variety crosses of maize. Z Pflanzenzuchtg, 83: 176-183.

Galal Y. A.A. (2014). Diallel analysis of some yellow maize inbred lines for yield and resistance to late wilt disease. Ph.D. Thesis, Fac. Agric. Kafr El-Sheikh Univ., Egypt.

Griffing, B. (1956). Concept of general and specific combining ability in relation to diallel crossing system. Australian J. Biol. Sci., 9: 463-493.

Kaneko, K. and B. A. Aday (1980). Inheritance of resistance to Philippine downy mildew of maize, Peronosclerospora philippinensis. Crop. Sci., 20: 590-594.

Melchers, L.E. (1931). Downy mildew of sorghum and maize in Egypt Phytopathology, 21: 239240.
Moradi, M. (2014). Combining ability for grain yield and some important agronomic traits in Maize (Zea mays L.). Int. J. Biosci., 5(4): 177185.

Mosa, H.E. and A.A. Motawei (2005). Combining ability of resistance to late wilt disease and grain yield and their relationships under artificial and natural infections in maize. J. Agric. Sci. Mansoura Univ., 30: 731-742.

Mosa, H.E.; A.A. Motawei and A.A. El-Shenawy (2009). Selection new single crosses of maize for grain yield and resistance to downy mildew disease under different location and potassium fertilization. J. Agric. Res. Kafr El-Sheikh Univ., 35(2) 2009.

Motawei, A.A. (2006). Gene action and heterosis in diallel crosses among ten inbred lines of yellow maize across various environments. Egypt. J. Plant Breed. 10(1): 407-418.

Murtadha, M.A.; O.J. Ariyo; S.S. Alghamdi (2016). Analysis of combining ability over environments in diallel crosses of maize (Zea mays). Journal of the Saudi Society of Agricultural Sciences.

Nair, S.K.; B.M. Prasanna; R.S. Rathore; T.A.S. Setty; R. Kumar and N.N. Singh (2004). Genetic analysis of resistance to sorghum downy mildew and rajasthan downy mildew in maize (Zea mays L. ) Field Crops Res. 89: 379387.

Nawar, A.A. and M.A. Salem (1985). Diallel analysis of inheritance of late wilt and leaf blight in maize. Minufiya J. Agric. Res. 10(2): 719-737.

Sabet, K.A.; A.M. Zaher, A.S. Samra, and I.M. Mansour (1970). Pathogenic behavior of Cephalosporium maydis and C. acremonium. Ann. App. Biol. 66:257-263.

Salama, S.I. (1976). Investigations of the major stalk, foliar and grain disease of sorghum (Sorghum bicolor) including studies on the genetic mature of resistance. Ann. Rep. Public Law, 480FGEG138.

Samra, A.S.; K.A. Sabet, and M.K. Hingorani (1962). A new wilt disease of maize in Egypt. Plant Dis. Rep. 46: 481-483.

Steel, R.G. and J. Torrie (1980). Principles and Procedures of Statistics. Mc Graw-Hill Book Company, New York, USA. 


\title{
الملخص العربي
}

\section{القدرة على التآلف والنسبة المئوية للتفوق في التهجين النصف دائري بين سلالات مرباه داخليا للأرة الصفر اء لصفات النمو والمقاومة لبعض الامر اض}

\author{
رمضان على الرفاعى1، عاصم عبده مطاوع²، أمجد عبدالغفار الجمال، محمد سعيد قطب2

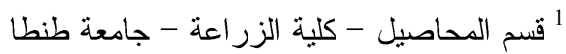

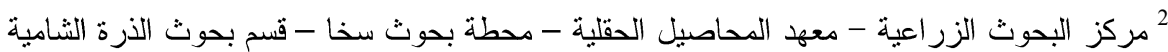

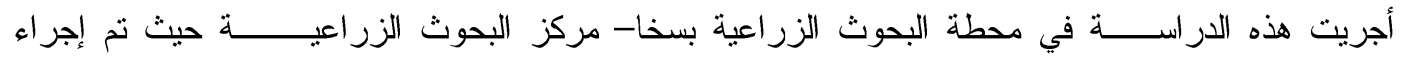

التهجين النصف تبادلي في موسم 2015 بين عشر سلالات مرباه داخليا للذرة الصفراء. تم تقييم الخمسة و أربعون

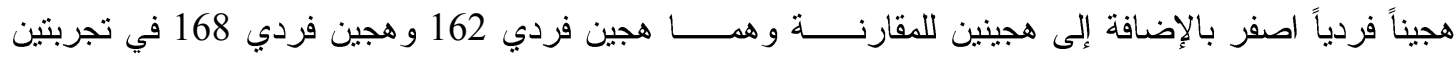

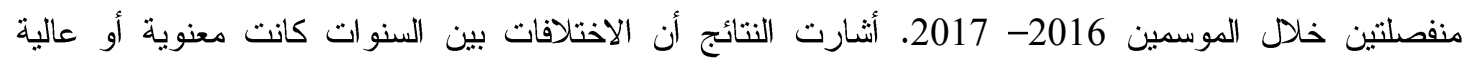
المعنوية لجميع الصفات محل الدراسة ماعدا المقاومة لمرض البياض الزغبي. كما أوضحت النتائج أن الاختلافات

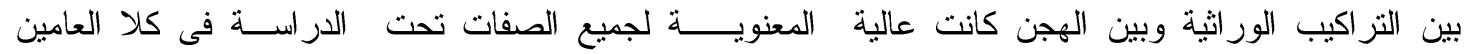
و التحليل المشترك بينما كان تفاعل كلا من التزر اكيب الور اثية و الهجن مع السنوات كان معنوي أو عالي المئ المعنوية

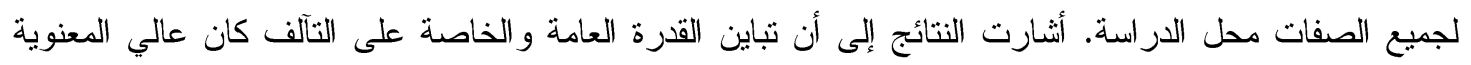
لجميع الصفات محل الدراسة فى كلا العامين والتحليل المشترك، كما أوضحت النتائج إلى أن تفاعل كلا من القدرة

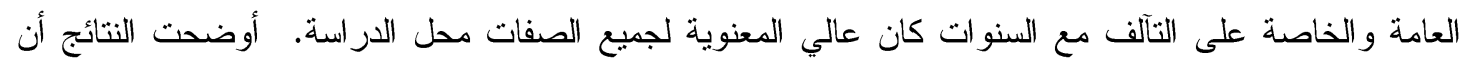

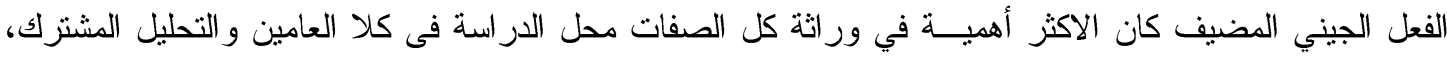
كما أوضحت النتائج أن الفعل الجيني المضيف كان الاكثر تفاعلا مع السنوات عن الفعل الجيني الغير مضيف فى في كل الصفات محل الدراسة فى كلا العامين و التحليل المشترك. أظهرت(السلالة 4) قدرة عامة على التآلف معنوية للتبكير ومواصفات النبات الجيد والمقاومة لمرضى البياض الزغبى و الذبول المتأخر، كما أظهرت(السلالة 8) قدرة

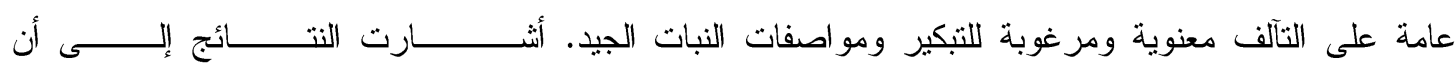

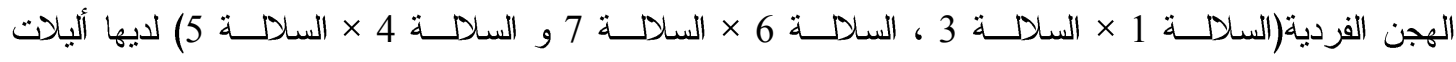

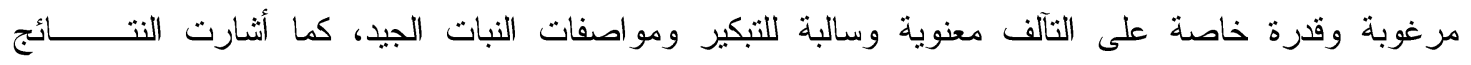

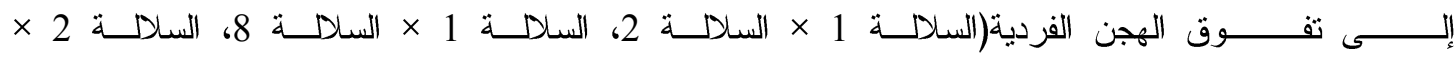

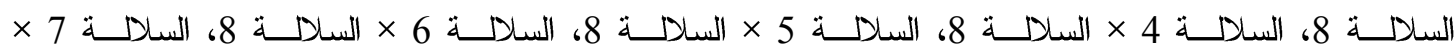

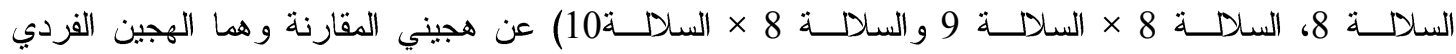
162 الهجين الفردي 168 للتبكير ومواصفات النبات الجيد. 